\title{
Water-soluble tocopherol derivatives inhibit SARS-CoV-2 RNA-dependent RNA polymerase
}

\author{
Hayden T. Pacl ${ }^{1}$, Jennifer L. Tipper ${ }^{2}$, Ritesh R. Sevalkar ${ }^{1}$, Andrew Crouse $^{3}$, Camerron \\ Crowder ${ }^{3}$, UAB Precision Medicine Institute ${ }^{3}$, Shama Ahmad², Aftab Ahmad², Gillian D. Holder²,

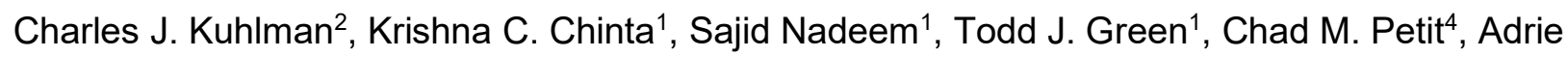 \\ J.C. Steyn ${ }^{1,5}$, Matthew Might ${ }^{3}$, Kevin S. Harrod ${ }^{2}$
}

${ }^{1}$ Department of Microbiology, School of Medicine, University of Alabama at Birmingham, Birmingham, AL USA

${ }^{2}$ Department of Anesthesiology and Perioperative Medicine, School of Medicine, University of Alabama at Birmingham, Birmingham, AL USA

${ }^{3}$ Precision Medicine Institute, School of Medicine, University of Alabama at Birmingham, Birmingham, AL USA

${ }^{4}$ Department of Biochemistry and Molecular Genetics, School of Medicine, University of Alabama at Birmingham, Birmingham, AL USA

${ }^{5}$ African Health Research Institute, University of KwaZulu Natal, Durban, South Africa

Running Title: TPGS antiviral activity against SARS-CoV-2

${ }^{*}$ Corresponding Author: Email: kevinharrod@uabmc.edu (K.S.H.) 
Author contributions: K.S.H. and M.M. oversaw conception and supervised the study. H.T.P., A.J.C.S., M.M., and K.S.H. designed the research. H.T.P., J.L.T., R.R.S., G.D.H., C.J.K., K.C.C., and S.N. performed the experiments. UAB PMI performed artificial intelligence screening and in silico drug identification. T.J.G. and C.M.P. performed the structural biology experiments. H.T.P., M.M. and K.S.H. wrote the paper. H.T.P. and K.S.H. designed and analyzed all experiments. A.J.C.S, M.M. and K.S.H provided financial support.

${ }^{3}$ UAB Precision Medicine Institute: Andrew Crouse, Camerron M. Crowder, Mae Crumbley, Aleksandra Foksinska, Matthew Jarrell, Tarun K. K. Mamidi, Lurong Pan, Michael J. Patton, Sienna Rucka, Nicholas T. Southern, Jillian Tinglin, Wesley Walters, Matthew Might

Acknowledgements: We thank the Southeastern Biosafety Laboratory (SEBLAB), an NIAID supported Regional Biocontainment Facility (NIAID UC6 AI058599) and leadership (Justin Roth, Chad Dunaway, Moustafa Awaden, and Simone Seay) for assistance in developing procedures and protocols in support of BSL3 studies of SARS-CoV-2. The authors would like to acknowledge Paul Wolkowitz, Joel Glasgow, Blake Frey, and Patrick Molina for their thoughtful feedback as well as for enabling the safe conduct of this research. Research reported in this publication was supported by the UAB High Resolution Imaging Facility, the UAB Heflin Genomics Core (P30CA013148), and the UAB SEBLAB Alabama Birmingham.

Funding: Supported by NIH grants R01HL149944 (KSH), R01Al134693 (CMP), U19 3U19AI142737 (TJG), T32HL13640 (HTP), Cystic Fibrosis Foundation grants HARROD19IO and HARROD20GO (KSH), UAB SOM COVID-19 Awards (CMP, MM, and KSH), Benjamin Monroe Carraway Endowment (KSH) 
Competing Interests: The authors' institution is pursuing a patent for the use of water-soluble tocopherol derivatives as antiviral compounds as described in this article.

Data Materials and availability: All drug compounds tested are available from Sigma Aldrich or other commercial entities. All relevant data are included in this manuscript and all materials are readily available upon request from the corresponding author. 


\section{Summary}

The recent emergence of a novel coronavirus, SARS-CoV-2, has led to the global pandemic of the severe disease COVID-19 in humans. While efforts to quickly identify effective antiviral therapies have focused largely on repurposing existing drugs ${ }^{1-4}$, the current standard of care, remdesivir, remains the only authorized antiviral intervention of COVID-19 and provides only modest clinical benefits ${ }^{5}$. Here we show that water-soluble derivatives of $\alpha$-tocopherol have potent antiviral activity and synergize with remdesivir as inhibitors of the SARS-CoV-2 RNA-dependent RNA polymerase (RdRp). Through an artificial-intelligence-driven in silico screen and in vitro viral inhibition assay, we identified D-a-tocopherol polyethylene glycol succinate (TPGS) as an effective antiviral against SARS-CoV-2 and $\beta$-coronaviruses more broadly that also displays strong synergy with remdesivir. We subsequently determined that TPGS and other water-soluble derivatives of $\alpha$-tocopherol inhibit the transcriptional activity of purified SARS-CoV-2 RdRp and identified affinity binding sites for these compounds within a conserved, hydrophobic interface between SARS-CoV-2 nonstructural protein 7 and nonstructural protein 8 that is functionally implicated in the assembly of the SARS-CoV-2 RdRp ${ }^{6}$. In summary, we conclude that solubilizing modifications to $\alpha$-tocopherol allow it to interact with the SARS-CoV-2 RdRp, making it an effective antiviral molecule alone and even more so in combination with remdesivir. These findings are significant given that many tocopherol derivatives, including TPGS, are considered safe for humans, orally bioavailable, and dramatically enhance the activity of the only approved antiviral for SARS-CoV-2 infection ${ }^{7-9}$.

\section{Introduction}

The emergence of multiple coronaviruses (CoVs) into the human population in recent years $^{10-12}$ highlights the need for the rapid development of broad-spectrum interventional strategies against genetically distinct CoVs. Despite the unprecedented rapid development of effective vaccines targeting the severe acute respiratory syndrome coronavirus 2 (SARS-CoV-2), 
the etiologic agent of COVID-19, breakthrough infections in the immunocompromised ${ }^{13-19}$, infections in the young and elderly lacking a full repertoire of immunological responses ${ }^{20}$, vaccine reticence or hesitancy ${ }^{21}$, and the inequitable distribution of vaccines globally ${ }^{22}$ indicate that therapeutic interventions will be required to limit severe disease and substantial mortality associated with pandemic SARS-CoV-2 infection. Currently, remdesivir, under emergency FDA authorization, is the only antiviral to show improvement of clinical outcomes, albeit with limited effectiveness and the limitation of intravenous administration ${ }^{5}$. Furthermore, breakthrough infections and viral evolution of resistant strains is possible ${ }^{14,23}$. This highlights the need for identifying new antivirals with broad efficacy against CoVs, and the potential for combinatorial interventional antivirals that reduce the development of resistance. Here, we employed a novel artificial intelligence query of FDA-approved compounds for repurposing as antivirals against SARS-CoV-2 and identify tocopherol derivatives with potent antiviral activity and synergy with remdesivir.

\section{Drug-repurposing against SARS-CoV-2}

To select candidates for in vitro screening, we employed the open-source artificial intelligence software mediKanren to perform a logical query of the existing literature for compounds that are known to induce or inhibit host proteins involved in reducing or enhancing viral replication, respectively (https://github.com/webyrd/mediKanren) (Fig S1). Complemented by a manual review of the available literature, we identified over 1300 compounds with proposed antiviral activity, of which nearly 100 were prioritized for in vitro screening (Table S1). To assess the antiviral activity of these compounds against virulent SARS-CoV-2, we adapted a traditional focus-forming assay in BSL-3 biocontainment by allowing foci to expand gradually over 48 hours, using progression of viral staining as a proxy for the magnitude of infection (Figure S2). Employing this method, we identified 12 compounds capable of reducing SARS-CoV-2 propagation by more than $90 \%$ at a concentration of $10 \mu \mathrm{M}$ (Figure 1A). Follow-up 
testing revealed these compounds exhibit reliable dose-response relationships within our antiviral assay that are independent of their toxicity profile (Figure 1B).

We expanded the characterization of candidate antiviral compounds by evaluation of antiviral activity across a range of conditions. We found that five of our top 12 compounds reduced the burden of SARS-CoV-2 in infected Calu3 cells, a human lung epithelial cell line, by greater than $90 \%$ (Figure 1C). Interestingly, four of these five drugs-niclosamide, remdesivir, lopinavir, and D-a-tocopherol polyethylene glycol succinate (TPGS)—were the only drugs to show maximum efficacy in halting an established SARS-CoV-2 infection in VeroE6 cells (Figure 1D). On the other hand, each of the top compounds except E-64d demonstrated strong antiviral activity against the seasonal $\beta$-coronavirus, OC43 (Figure 1E; Figure S3), suggesting that the majority of the compounds we identified are effective against $\beta$-coronaviruses more broadly. Overall, these findings describe four compounds with robust, broad-spectrum antiviral activity and therapeutic applications.

\section{TPGS synergizes with remdesivir}

Given the threat of drug-resistance evolving in response to single-agent therapy, as well as the modest effects and limited supply of currently available antivirals for the treatment of COVID-19, we screened our top compounds for synergistic combinations. Relying on the principle of Loewe Additivity ${ }^{24,25}$, we combined our top compounds in a pairwise fashion to yield equipotent mixtures with a predicted efficacy of $50 \%$. While the myriad combinations revealed mostly additive effects, the combination of remdesivir and TPGS resulted in an 8-fold increase in potency (Figure 2A). Follow-up testing of equipotent combinations of the top drugs with either remdesivir (Figure 2B; Figure S4) or TPGS (Figure 2C) revealed that this combination was indeed unique and doseresponsive across a range of concentrations. To fully characterize the synergy between TPGS and remdesivir we expanded our analysis across a range of mixtures to perform response-surface analysis, an extension of traditional isobologram analysis ${ }^{25}$. We identified that an equipotent 
mixture of TPGS and remdesivir was optimal for antiviral activity (Figure 2D, E), and, at this ratio, we observed a reduction of approximately 10 -fold in the $\mathrm{IC}_{90}$ (Figure $2 \mathrm{E}$ ). Of note, including one drug as at least $10 \%$ of the total combined dose dramatically enhances the potency of the other, suggesting that synergism can be honed to limiting availability to either compound.

\section{$\alpha$-Tocopherol drives TPGS activity}

Given the pronounced synergy between remdesivir and TPGS, we next sought to understand the mechanism for the antiviral activity of TPGS, starting with its known, biologicallyrelevant activities: a non-competitive inhibitor of the drug exporter P-glycoprotein (PGP) ${ }^{26,27}$, a surfactant, and a derivative of Vitamin E. Upon querying our list of screened drugs for substrates or inhibitors of PGP, we found no clear relationship between substrates (i.e. competitive inhibitors) of PGP and antiviral activity (Figure S5A). Additionally, we further screened well-characterized inhibitors and inducers of PGP for antiviral activity against SARS-CoV-2 (Figure S5B-G). While the majority showed no effect, amiodarone, another non-competitive inhibitor of PGP, exhibited antiviral activity against SARS-CoV-2 (Figure S5G). Amiodarone, however, showed no ability to synergize with remdesivir (Figure $\mathrm{S} 4 \mathrm{H}$ ), suggesting that the antiviral activities of TPGS and amiodarone are mediated by independent mechanisms and are therefore unlikely to be related to their commonality as PGP inhibitors. Additionally, TPGS is a surfactant. Other surfactant molecules with related chemical structures showed no antiviral activity (Figure S5I-L).

Finally, we tested the constituent components of TPGS: D- $\alpha$-tocopherol, succinate, and polyethylene glycol with a molecular weight of 1000 Da (PEG-1000; Figure S6A). a-tocopherol alone is insoluble in an aqueous environment, so we instead tested $\alpha$-tocopherol succinate

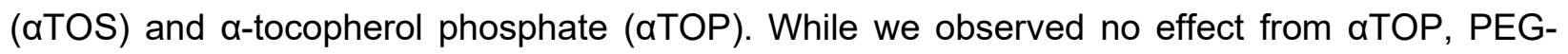
1000 , or succinate alone (Figure S6B-D), we found that aTOS is capable of inhibiting SARS-CoV2 replication in VeroE6 cells, albeit at higher concentrations than TPGS $\left(\mathrm{IC}_{50}=21.7 \mu \mathrm{M}\right.$; Figure 
S5E). These findings suggests that a-tocopherol is the active component of TPGS, and succinate and PEG-1000 contribute indirectly to its potency.

To determine whether the synergy with remdesivir and the increased potency of TPGS was specific to PEG-1000 or limited to the D-isomer of a-tocopherol, we tested a related compound: DL-a-tocopherol methoxypolyethylene glycol succinate (TPGS-750-M). TPGS-750-M is composed of an a-TOS molecule modified with a shorter PEG that terminates in a methoxy group, and the DL indicates that this compound consists of a racemic mixture of equal parts of the eight possible stereoisomers of a-tocopherol. Despite these differences, TPGS-750-M demonstrated comparable antiviral activity to TPGS (Figure S6F) as well as the ability to synergize with remdesivir (Figure S6G,H). Within logical constraints, these data suggest that medicinal chemistry targeting the size of the PEG or the isomer of the a-tocopherol may be modified to yield a compound with different physical and chemical properties that retains its antiviral activity.

\section{Tocopherols inhibit SARS-CoV-2 RdRp}

We next sought to determine where in the process of viral propagation TPGS acts. We therefore infected VeroE6 cells with SARS-CoV-2 for 30 minutes and measured both intracellular and extracellular SARS-CoV-2 genome expression at 0, 3, and 9 hours post infection (hpi). We observed no decrease in viral attachment or uptake, but we detected a $\sim 4000$-fold reduction in genomic material at $9 \mathrm{hpi}$ in cells treated by either remdesivir or TPGS (Figure 3A). Notably, there was no detectable extracellular genome at any of these timepoints, indicating inhibition of viral transcriptional activity alone (data not shown).

We expanded our analysis of the expression of SARS-CoV-2 RNA by sequencing mRNA from the samples in our time course experiment and analyzing the transcriptome of infected host cells. We observed the same profound reduction in transcripts mapped to each of the genes in 
the SARS-CoV-2 genome and detected no RNA corresponding to many of the viral genes in our treated samples (Figure S7A). The latter point, in particular, suggests that TPGS inhibits the transcription of viral genes. With regard to the transcription of host mRNA, principle component analysis revealed treated and untreated samples at 9 hpi have distinct transcriptomes (Figure S7B). Follow up gene ontology analysis of TPGS-treated and untreated cells at 9 hpi revealed that of the top 20 biological processes identified as enriched, ten were related to lipid metabolism (Figure S7C). The latter suggests that TPGS-treated cells respond to the tocopherol component of TPGS.

Considering the potent synergy between TPGS and remdesivir, a known inhibitor of the SARS-CoV-2 RNA-dependent RNA polymerase $(\operatorname{RdRp})^{28,29}$, as well as the results from our time course experiment, we hypothesized that TPGS inhibits the SARS-CoV-2 RdRp. To directly test our hypothesis, we measured the ability of TPGS to inhibit the transcriptional activity of purified SARS-CoV-2 RdRp composed of the catalytic subunit NSP12, and two accessory proteins, NSP7 and NSP8. Indeed, TPGS is a potent inhibitor of the transcriptional activity of the SARS-CoV-2 $\operatorname{RdRp}\left(\mathrm{IC}_{50}=212 \mathrm{nM}\right.$; Figure 3B $)$-approximately 100-fold more potent than the active metabolite of remdesivir (RTP; IC $50=26 \mu \mathrm{M}$; Figure 3B).

Interestingly, we also observed that TPGS does not inhibit RdRp at concentrations above its critical micelle concentration (CMC; Figure $3 C)^{30}$. This led us to the expanded hypothesis that water-soluble derivatives of $\alpha$-tocopherol inhibit the SARS-CoV-2 RdRp. We tested this hypothesis using aTOS and $\alpha \mathrm{TOP}$ in our RdRp transcriptional assay. Consistent with our results in VeroE6 cells, aTOS was capable of inhibiting transcription; however, we also observed comparable inhibition of the SARS-CoV-2 RdRp by aTOP (Figure 3D). These findings suggests that while the efficacy of these compounds as antivirals may vary in the more complex cellular environment, water-soluble tocopherols inhibit the SARS-CoV-2 RdRp. 
Finally, to further elucidate the interaction between water-soluble tocopherols and the SARS-CoV-2 RdRp, a series of computational docking studies were performed. Due to the challenge of modeling the flexibility of the PEG in the TPGS molecule as well as the ability of aTOS to inhibit the purified RdRp as well as viral replication in cells, we performed these studies for aTOS. Our docking studies across the individual components of the RdRp identified high affinity binding sites for aTOS within each NSP7, NSP8, and NSP12 (Figure 3E,F; Figure S8). Interestingly, while the top poses for aTOS were spread to a few locations across the surface of NSP12, our docking studies on the interaction of aTOS with NSP7 and NSP8 each converged to a single region (Figure S8A-C). In each case, the most energetically favorable binding poses localized with residues that participate in one of the two recently described hydrophobic interfaces that is required for the assembly of the functional RdRp (Figure 3G; Figure S8D) ${ }^{6}$. The residues within this interface are highly conserved across coronaviruses, and site directed mutagenesis of several residues within this interface reduced or ablated the transcriptional activity of the SARSCoV-2 RdRp 6 . Taken together, these findings strongly support a mechanism by which TPGS prevents or destabilizes the assembly of the SARS-CoV-2 RdRp.

\section{TPGS promotes RNA capping by RdRp}

Since TPGS is an inhibitor of the SARS-CoV-2 RdRp, we next hypothesized that the synergy of TPGS and remdesivir stems from independent mechanisms of inhibition of transcriptional activity. We combined TPGS and RTP at concentrations that independently led to intermediate inhibition of transcription in our RdRp assay and determined the combination index of these mixtures. Contrary to our hypothesis, we observed a purely additive combinatorial effect (Figure 4A). This suggests that the synergy between these compounds stems from a mechanism beyond the inhibition of transcription alone.

Given these results and the recent description of remdesivir as an inhibitor of the guanylyl transferase (GTase) activity of NSP12 ${ }^{31}$, we next decided to test GTase activity of NSP12 in the 
presence of TPGS. Compared to untreated, TPGS enhanced the GTase activity of the NSP12 in vitro (Figure 4B,C). This suggests that in the presence of TPGS, the RdRp function of NSP12 is inhibited, though the alternate GTase function of NSP12 is not. However, in the presence of both TPGS and remdesivir, both known activities of NSP12-essential for the production of mature viral RNA-are potently inhibited (Figure 4D). Blocking both distinct enzymatic functions could yield the observed synergy.

\section{Discussion}

Here, we have identified water-soluble derivatives of tocopherol as inhibitors of the SARSCoV-2 RdRp. Importantly, the most potent of these tested, TPGS, synergizes with remdesivir to yield a highly effective combination therapy. Docking studies suggest that tocopherol derivatives destabilize or inhibit the assembly of the SARS-CoV-2 RdRp by binding to hydrophobic residues at a highly conserved interface between NSP7 and NSP8 required for the assembly of the functional RdRp formed by NSP12, NSP7, and NSP8 ${ }^{6}$.

While there are sites on the surface of NSP12 with marginally higher affinity for aTOSmost notably at the hydrophobic interface between NSP8 and NSP12-(Figure S8E), all top poses for the models of aTOS interacting with NSP7 and NSP8 localize to a single site within each protein, providing greater confidence in their relevance. This mechanism is further supported by the knowledge that these two sites together form a single interface required for the assembly of the functional SARS-CoV-2 RdRp ${ }^{6}$. Interestingly, this mechanism would explain our observation that TPGS treatment enhances the GTase activity of NSP12 in the presence of NSP7 and NSP8, as the disruption of the assembled RdRp complex in this assay would prevent NSP12 from participating in the assembled RdRp, thereby freeing all NSP12 to participate in 5' capping.

We consider water-solubility a key factor in the potency of these compounds. Regardless of the mechanism, inhibition of the polymerase complex would require the tocopherol molecules 
to exist in a soluble form within the aqueous environment of the cell. Pegylation is a known method of solubilizing small molecules, and we attribute the considerable improvement in potency to PEG derivatization in TPGS when compared to aTOS. A corollary of this argument is that the antiviral activity of these compounds in a biological context relies on the stability of the solubilizing modification to the tocopherol which varies in biological systems ${ }^{32}$. Should the modification be hydrolyzed, the hydrophobic tocopherol will not remain in solution and will lose its antiviral effect. This may account for the variable efficacy of antiviral activity between $\alpha$ TOS and $\alpha$ TOP in viral replication assays (Figure S6D,E), despite comparable efficacy and potency in our transcriptional activity assay (Figure 3D). It is likely that both stability in an aqueous environment and hydrophobicity leading to greater cellular uptake both contribute to the antiviral activity of tocopherol derivatives tested here.

These considerations along with a vast literature highlight the potential of this group of molecules for optimization as clinical therapeutics. TPGS micelles have been studied extensively as a tool to enhance the solubility, oral-bioavailability, and the tissue penetration of numerous drugs $^{9,33-36}$. The critical micelle concentration of TPGS, as well as its capacity for PGP inhibition, can be shifted by modifying the length of the attached PEG molecule 37,38 . The biological activity of Vitamin $\mathrm{E}$ is affected by its stereochemistry ${ }^{39-41}$, while its antiviral activity is not (Figure S6F). Finally, further solubilizing modifications should be explored to optimize solubility, potency, and stability, as well as the exploration of related tocopherols and isoprenoids. In combination or isolation, these characteristics can be modified to maximize antiviral efficacy while limiting potential host-directed effects of this direct-acting antiviral compound.

Though remdesivir is known to be a non-obligate terminator of transcription effective against SARS-CoV-2 and other viruses ${ }^{28,29,42}$, this activity alone does not account for the observed synergy-and therefore complete antiviral activity_observed when in combination with TPGS (Figure 4A). We note that remdesivir was recently described as an inhibitor of the GTase activity 
of NSP12 ${ }^{31}$. TPGS does not inhibit this reaction; rather, the GTase activity of NSP12 is shown to be potentiated by TPGS (Figure 4B,C). We propose a model where TPGS and remdesivir synergize by inhibiting two sequential enzymatic reactions in the SARS-CoV-2 transcription cycle: polynucleotide synthesis and mRNA-capping, both of which are essential for cap-dependent translation in mammalian cells ${ }^{43}$. Presumably, both compounds also inhibit the two-step process of viral genomic replication, (+) and (-) strand synthesis of the coronavirus genome. Taken together, it is conceivable that this forms the conceptual framework for TPGS and remdesivir synergy. This model also provides an explanation for the consistent observation that the $\mathrm{IC}_{50}$ for the antiviral activity of remdesivir is orders of magnitude lower than the $\mathrm{IC}_{50}$ for the inhibition of the transcriptional or capping activity ${ }^{2,28,44-46}$. It follows, then, that the potency of remdesivir as an antiviral against SARS-CoV-2 may stem from its combined activity against both processes which can be enhanced by more potent transcriptional inhibition from TPGS.

In summary, we have identified a stable, safe, and orally-bioavailable compound, TPGS, that synergizes with the current standard of care, remdesivir, to inhibit SARS-CoV-2 replication. These findings also argue that inhibition of the GTase activity of NSP12 is an important part of the antiviral activity of remdesivir. Lastly, the antiviral activity of TPGS extends to a larger class of bioactive molecules with unique biophysical characteristics that retain their antiviral activity with chemical modification, opening the door to a new class of antiviral drugs.

\section{Methods}

\section{Cell lines}

Stocks of low-passage VeroE6 (ATCC \# C1008) and Calu3 (ATCC \# HTB-55) cells were stored in liquid nitrogen. As needed, stocks were revived and grown to confluency in a tissueculture-treated flask at $37^{\circ} \mathrm{C}$ and $5 \% \mathrm{CO}_{2}$ in complete minimal essential media (MEM) (Gibco \# 11430-030) containing 10\% fetal bovine serum (Denville Scientific Inc \# C788U20); 2 mM 
glutamine (Gibco \#25030-081); an antibiotic/antimycotic cocktail of peniciliin (100 units / $\mathrm{mL}$ ), streptomycin (100 units / mL), and amphotericin B (250 ng / mL) (Gibco \#15240-062); $25 \mathrm{mM}$ HEPES (Gibco 15630-080); and 25 mM sodium bicarbonate (Gibco \# 25080-094). At confluency, cells were detached using $0.25 \%$ trypsin-EDTA (Gibco \# 25200-056), and seeded in a tissueculture treated, 96-well microplate at a density of $4 \times 10^{4}$ cells/well or dispensed to a flask (1:3) for maintenance. VeroE6 cells were seeded the day prior to experiments, and Calu3 cells were seeded four days prior to experiments.

\section{Virus}

SARS-CoV-2 (Wa-1/USA) virus was obtained from BEl and propagated in VeroE6 cells. Virus was harvested from the cell culture supernatant by centrifugation at $\sim 1700 \times \mathrm{g}$ for 5 minutes to remove cell debris and subsequent collection of supernatant containing virus. The resulting preparation was aliquoted and stored at $-80^{\circ} \mathrm{C}$. All experiments in this manuscript were performed with third-passage or fourth-passage SARS-CoV $-2\left(10^{7}\right.$ plaque forming units $\left./ \mathrm{mL}\right)$. Sequence verification of SARS-CoV-2 WA-1/USA at each passage was performed with less than 10 synonymous invariants observed in each passage, and none in critical regions of the polymerase complex genetic loci.

\section{Drug Preparation and Storage}

Information regarding the drugs used in screening experiments is available in Table S1. Where possible, drugs were prepared in $100 \%$ DMSO at a concentration of $20 \mathrm{mM}$, aliquoted to minimize freeze/thaw cycles, and stored at $-20^{\circ} \mathrm{C}$. If they were insoluble in DMSO, drugs were prepared in tissue culture grade water (Cytiva/Hyclone \# SH30529.03). Drugs used in screening experiments were purchased from Millipore Sigma. PGP inhibitors and inducers in Figure S4 were purchased from Cayman Chemicals. Surfactants in Figure S4 were purchased from Millipore 
Sigma. The active metabolite of remdesivir, GS-443902, was purchased from Med Chem Express and stored at $-80^{\circ} \mathrm{C}$ for a maximum of one month.

\section{Artificial-Intelligence-Driven Screen}

The artificial intelligence tool mediKanren was used to efficiently identify FDA-approved, readily available drug compounds hypothesized to target human and/or viral proteins essential for SARS-CoV-2 infection for further screening in vitro (Figure S1). MediKanren is a biomedical reasoning system composed of a custom knowledge-graph database and a constraintbased logic reasoning engine, developed from the miniKanren family of relationalreasoning logic languages ${ }^{47}$. The reasoning engine finds biological relationships between biomedical concepts, such as molecules, drugs, proteins, pathways, genes, diseases, symptoms, and phenotypes and can identify candidates that are directly or indirectly connected. Its knowledge graph database incorporates over 180 distinct sources of biomedical knowledge, including the scientific literature (Pubmed), data on approved and unapproved drugs (via FDA and drugbank), and genes (NCBI, UniProt). This initial computational screen for human and/or viral proteins related to SARS-CoV infection produced approximately 1,300 compounds that were algorithmically prioritized and then manually investigated based on hypothesized therapeutic benefit. MediKanren was developed by the Hugh Kaul Precision Medicine Institute at UAB and source code for mediKanren software is available free of charge as open source on github (https://github.com/webyrd/mediKanren).

\section{Antiviral activity assay}

One hour prior to infection, unless otherwise indicated, cells were washed once in serumfree (SF) MEM and incubated in SF-MEM containing the indicated treatment. Infections were 
performed for one hour in SF-MEM at $35^{\circ} \mathrm{C}$ at a multiplicity of infection (MOI) of 0.1 for SARSCoV-2 in VeroE6 cells, an MOI of 1 for SARS-CoV-2 in Calu3 cells, and an MOI of 0.1 for OC43 in VeroE6 cells. Following the infection, an equal volume of media containing Avicel (FMC BioPolymer \# Avicel CL-611) and fetal bovine serum was added to achieve a final concentration of $0.6 \%$ and $2 \%$, respectively. Cells were then incubated at $35^{\circ} \mathrm{C}$ for 48 hours and fixed in $10 \%$ phosphate buffered formalin for a minimum of 1 hour and a maximum of 72 hours.

Following infection and fixation, cells were immunostained for SARS-CoV-2 spike protein. First, endogenous peroxidase activity in fixed monolayers was blocked by incubation in methanol containing $0.5 \%$ hydrogen peroxide for 30 minutes. Following three washes in deionized water, nonspecific protein binding was blocked by incubation in phosphate-buffered saline (PBS) containing $5 \%$ nonfat milk for 20 minutes. Cells were then directly incubated with a rabbit polyclonal antibody against SARS-CoV-2 spike/RBD protein (SinoBiological \# 40592-T62), diluted 1:2500 in milk on a rocking platform for 1 hour at room temperature. Following this incubation, cells were washed five times with PBS and then incubated with horseradishperoxidase-conjugated goat-anti-rabbit secondary antibody (Invitrogen \# A16104) diluted 1:1000 in milk shaking for 1 hour. Cells were washed once in PBS containing $0.05 \%$ tween 20 and again five times with PBS. Finally, cells were incubated for $3-12$ minutes with DAB reagent (Vector Laboratories), washed five times in water, and dried overnight while protected from light. For OC43, an identical procedure was followed, except that the primary antibody for viral detection was a monoclonal antibody against the coronavirus group antigen (Milipore Sigma \#MAB9013) and the secondary was a horseradish-peroxidase-conjugated goat-anti-mouse antibody (enQuire BioReagents \# Q2AB1).

Brightfield images of whole wells were acquired using either the Cytation 5 Cell Imaging Multi-Mode Reader (BioTek) or the Lionheart FX Automated Microscope (BioTek). Both instruments were operated with Gen5 software (BioTek), and images were acquired with the 
lowest power objective available-4x for the Cytation 5 and $1.25 x$ for the Lionheart FX, respectively. Focusing was performed with the dual-peak (brightfield) method and stitching of images within a well was performed using the linear-blend method. Whole-well images were then processed in FIJI software ${ }^{48}$ where they underwent automated well-detection by thresholding and particle analysis followed by gaussian filtering, background flattening, and thresholding of the original image to determine the proportion of the well stained for viral protein.

The percentage of area determined to be positive for viral protein within each well was normalized to the mean value calculated across all control wells within a given experiment. This resulted in a standardized measurement of "viral burden"-a near-normally distributed variable with a high degree of concordance between replicates and experiments (Figure S2).

\section{Viability Assay}

Uninfected VeroE6 cells were otherwise treated under the indicated condition as described in the antiviral activity assay. At 48 hours of treatment, cells were washed once in PBS and stained with Hoescht 33342 (ThermoFisher Scientific)—a membrane permeable nuclear stain-at $2 \mu \mathrm{g} / \mathrm{mL}$ and Sytox Green (ThermoFisher Scientific)—a membrane impermeable nuclear stain—at $1 \mu \mathrm{M}$ for twenty minutes. Cells were washed three times with PBS and placed in PBS for imaging.

Automated imaging and analysis was performed using the Lionheart FX and Gen5 software. Hoescht 33342 and Sytox green staining was imaged with the 4x objective in two independent fields of view for each well, using the DAPI and GFP filter cubes, respectively. Acquired images were processed in Gen5 software by background flattening and blurring prior to nuclear segmentation based on Hoescht 33342 staining. Nuclear segmentation was performed by thresholding and watershed separation of touching objects. Nuclei below a conservative threshold for mean fluorescent intensity of Sytox green staining were considered live cells, and 
viability was calculated as a proportion of live cells to total cells within a well. $2000-4000$ nuclei were analyzed per well.

\section{Statistical Analysis}

All statistical analyses were performed with $R$ statistical software (version 4.0 .2$)^{49}$ in the RStudio integrated development environment (version 1.3) ${ }^{50}$. Data were organized and graphed with the "tidyverse" collection of packages ${ }^{51}$, with the exception of response-surface analyses, which were performed with the "plotly" package ${ }^{52}$. Dose-response models predicting viral burden across a range of concentrations for a given intervention were calculated using the DRM function from the "DRC" package ${ }^{53}$. A four-parameter log-logistic model (equation 1) was used to fit viral burden to treatment dose:

$$
\text { 1) } f(x,(b, c, d, e))=c+\frac{d-c}{1+e^{b(\ln (x)-\ln (e))}}
$$

where $b$ corresponds to the steepness of the curve, $c$ is the lower asymptote of the curve, $d$ is the upper asymptote of the curve, and $e$ indicates the dose yielding a $50 \%$ effect. Because we operationally defined the untreated well as $100 \%$ viral burden and we modeled dose-response relationships only for drugs capable of suppressing viral burden to our limit of detection, $c$ and $d$ were fixed at 0 and 100, respectively, unless otherwise indicated. Gene Ontology analysis was performed with the "goseq" package as previously described ${ }^{54}$.

\section{Synergy analysis}

The framework of Loewe additivity was applied for all assessments of synergy ${ }^{24}$. In brief, Loewe additivity relies on the dose equivalence principle—the dose of drug $A$ that yields a given effect is equivalent to the dose of drug B that yields the same effect-and the principle of sham additivity-a drug added to itself is a purely additive effect ${ }^{25}$. Using the dose-response model for 
each drug (Figure 1B), we calculated the dose of the combined drugs in terms of IC50 equivalents as shown in equation $2^{55}$ :

$$
\text { 2) } I C_{50} \text { equivalent concentration }=\frac{A_{\chi}}{A_{50}}+\frac{B_{\chi}}{B_{50}}
$$

where $A_{x}$ is the concentration of drug A that yields effect $x, A_{50}$ is the $\mathrm{IC}_{50}$ for $\operatorname{drug} \mathrm{A}, B_{x}$ is the concentration of drug $\mathrm{B}$ that yields effect $x$, and $B_{50}$ is the $\mathrm{IC}_{50}$ for drug $\mathrm{B}$.

For synergy screening, individual drugs were prepared at a concentration of $1 \mathrm{IC}_{50}$ equivalent. Each compound was then mixed 1:1 with each other drug in a pairwise fashion, including itself (additive control), resulting in a mixture containing $0.5 \mathrm{IC}_{50}$ equivalents of Drug $\mathrm{A}$, $0.5 \mathrm{IC}_{50}$ equivalents of Drug $\mathrm{B}$, and a total dose of $1 \mathrm{IC} \mathrm{C}_{50}$ equivalent. By definition, a purely additive combination of drugs given at this dose yields a $50 \%$ reduction in the viral burden for that well. Based on the principle of sham additivity, observed effects in additive control wells were used to determine the true dose administered for each drug in each experiment, and the expected additive effect for each mixture was adjusted accordingly. Given the potential for doses corresponding to the upper and lower portions of a dose response curve to lead to incorrect conclusions of antagonism and synergy, respectively ${ }^{25,56}$, if the additive control for the given drug was outside of the linear range of the dose response curve (concentrations yielding approximately $20 \%-80 \%$ efficacy), all combinations of drugs containing that drug were excluded from analysis within that experiment.

For curve shift analysis, a similar approach to that described for synergy screening was used. In brief, drugs prepared at a concentration of $4 I C_{50}$ equivalents were combined $1: 1$ with another drug, resulting in an equipotent mixture of two drugs with a total dose of 4 IC50 equivalents. The resulting mixture was then serially diluted to yield a range of concentrations above and below the predicted $\mathrm{IC}_{50}$. 
For isobologram and response surface analysis, the approach to curve shift analysis was expanded to encompass a range of potency ratios, also known as a fixed-ratio or "ray" design, as shown in equation $3^{57}$ :

3) $Z=f A_{50}+(1-f) B_{50}$

where $f$ was increased from 0 to 1 by 0.1 . This yields a series of mixtures, $Z$, ranging from $0 \%$ drug $A$ and $100 \%$ drug $B$ to $100 \%$ drug $A$ and $0 \%$ drug $B$ by $10 \%$ increments. Given that the potency ratio between two drugs may vary above and below their respective $\mathrm{IC}_{50}$ values, we represented the additive isobole for the $\mathrm{IC}_{90}$ as a curvilinear isobole, as shown in equation $4^{58}$ :

$$
\text { 4) } b=B_{90}-B_{50}\left(\frac{a}{A_{50}}\right)^{\frac{q}{p}}
$$

where $B_{90}$ is the $\mathrm{IC}_{90}$ for drug $\mathrm{B}, B_{50}$ is the $\mathrm{IC}_{50}$ for $\operatorname{drug} \mathrm{B}$, a is a given concentration of drug $\mathrm{A}$, $A_{50}$ is the $\mathrm{IC}_{50}$ for drug $\mathrm{A}, \mathrm{q}$ is the slope parameter calculated for the dose-response model of $\operatorname{drug} B$, and $p$ is the slope parameter calculated for the dose-response model of $\operatorname{drug} A$.

\section{Viral uptake and replication assay}

VeroE6 cells in a 6-well plate were infected with SARS-CoV-2 at an MOI of .1 for 30 minutes. At this point, considered $0 \mathrm{hpi}$, virus was removed, and cells were washed thoroughly with PBS three times and returned to complete MEM. At the indicated time points, cells were lysed and RNA was isolated using the Qiagen RNeasy minikit (Qiagen). RNA concentration was determined for each sample using a micro-drop spectrophotometer/fluorometer (DeNovix model DS-11 Fx +). The abundance of SARS-CoV-2 genetic material was subsequently determined by quantitative PCR using a Taqman probe and primers (Biosearch Technologies). The primers and probe sequences for the were as recommended by BEI resources for compatability with synthetic RNA standards (\# NR-52358).

\section{mRNA sequencing and analysis}


mRNA-sequencing was performed on the Illumina NextSeq500 as described by the manufacturer (Illumina Inc., San Diego, CA). Briefly, RNA quality was assessed using the Agilent 2100 Bioanalyzer. RNA with a RNA Integrity Number (RIN) of $\geq 7.0$ was used for sequencing library preparation. RNA passing quality control was converted to a sequencing ready library using the NEBNext Ultra II Directional RNA library kit with polyA selection as per the manufacturer's instructions (NEB, Ipswich, MA). The cDNA libraries were quantitated using qPCR in a Roche LightCycler 480 with the Kapa Biosystems kit for Illumina library quantitation (Kapa Biosystems, Woburn, MA) prior to cluster generation.

TrimGalore!

(version

(https://www.bioinformatics.babraham.ac.uk/projects/trim_galore/) was used to trim the raw sequence FASTQ reads of primer adapter contamination (parameters used: --paired --trim-n -trim1 --nextseq 20). STAR (version 2.7.7a) was used to align the trimmed FASTQ reads to the combined Vero and SARS-CoV-2 Washington strain genomes (parameters used: -outReadsUnmapped Fastx --outSAMtype BAM SortedByCoordinate --outSAMattributes All) ${ }^{59}$. Following alignment, HTSeq-count (version 0.13.5) was used to count the number of reads mapping to each gene (parameters used: -m union -r pos -t exon -i gene_id -a 10 -s no -f bam) ${ }^{60}$.

\section{Transcriptional Activity of SARS-CoV-2 RNA-dependent RNA polymerase}

The transcriptional activity of the SARS-CoV-2 RdRp was assessed using the SARS-CoV2 RNA-dependent RNA polymerase kit plus (Profoldin \#S2RPA100KE), according to the kit instructions for reactions in a 384-well plate. In brief, reactions were carried out at $35^{\circ} \mathrm{C}$ for 2 hours in a total of $25 \mu \mathrm{L}$ in the presence of the indicated treatment condition. $65 \mu \mathrm{L}$ of fluorescent dye were added and fluorescence was determined on a per-well basis in a Synergy H1 multimode plate reader (BioTek). 0\% transcriptional activity was defined by the no-enzyme control and $100 \%$ activity was defined by an untreated control. 


\section{Docking Studies}

Docking experiments were performed using Autodock Vina ${ }^{61}$. Individual NSP7 and NSP8 structures were isolated from the structure of the SARS-CoV-2 NSP7-NSP8 complex (PDB ID: 7JLT $)^{6}$ while SARS-CoV-2 NSP12 was isolated from the SARS-CoV-2 RNA-dependent RNA polymerase structure (PDB ID: 6M71) ${ }^{62}$. These structures and a-tocopherol succinate were modeled in Autodock Tools with a-tocopherol succinate possessing 16 rotatable bonds. The search space was defined as a $36.75 \times 38.25$ x $37.50 \AA$ box for NSP7, a $48.75 \times 52.50 \times 41.25 \AA$ box for NSP8, and an $82.50 \times 89.25 \times 88.12 \AA$ box for NSP12 and encompassed the entire structure as to not bias the docking. No residues were designated as flexible in any of the receptors. The ten lowest energy solutions were then analyzed, and figures were generated in Pymol using a combination of the pose with the lowest energy as well as all poses found. Autodock Vina employs a strategy of initiating multiple runs starting from random ligand conformations. Free energy of binding calculations are performed using the intermolecular component of the lowest-scoring conformation as calculated via AutoDock Vina's scoring function $^{61}$.

\section{NSP12 Guanylyl Transferase Activity Assay}

The GTase activity assay was performed as previously described ${ }^{45}$. To generate diphosphorylated RNA for the GTase activity assay, $5 \mu \mathrm{M}$ of 20-base model RNA (5'AAUCUAUAAUAGCAUUAUCC-3'; ThermoFisher Scientific) was treated with $300 \mathrm{nM}$ of purified recombinant SARS-Cov2 NSP13 with an N-terminal His-tag (Cayman Chemicals \#30589) in the presence of $50 \mathrm{mM}$ Tris $\mathrm{pH}-7.4,5 \mathrm{mM} \mathrm{MgCl}$, and $10 \mathrm{mM} \mathrm{KCl}$ for 15 mins at $30^{\circ} \mathrm{C}$, followed by heat inactivation of $\mathrm{NSP} 13$ at $70^{\circ} \mathrm{C}$ for 5 mins.

The GTase reaction was carried out by incubating $500 \mathrm{nM}$ of SARS-Cov-2 RNAdependent RNA polymerase (complex of NSP12, NSP7 and NSP8; ProFoldin \#RDRP-100S2) 
with $1 \mu \mathrm{M}$ diphosphorylated 20 mer RNA in the presence of $0.05 \mu \mathrm{M}$ of $\alpha-32 \mathrm{P}-\mathrm{GTP}$ (PerkinElmer \#BLU006H), $5 \mathrm{mM} \mathrm{MgCl}$, and $10 \mathrm{mM} \mathrm{KCl}$ for 240 mins at $30^{\circ} \mathrm{C}$. A positive control GTase reaction using $0.5 \mathrm{U} / \mu \mathrm{L}$ of the vaccinia capping enzyme (New England Biolabs \#M2080S) in place of the SARS-CoV-2 RdRp was performed under similar conditions. The reaction was stopped by addition of loading dye consisting of $80 \%$ formamide, $10 \mathrm{mM}$ EDTA, $0.01 \%$ Bromophenol Blue, $0.02 \%$ xylene Cyanol and $15 \%$ glycerol. The reaction products were resolved on $15 \%$ denaturing PAGE gel with $7 \mathrm{M}$ Urea (Bio-Rad \#3450091), and was exposed to a phosphor screen (Amersham) overnight. The phosphor screen was scanned on the Typhoon FLA-7000IP (GE Healthcare). Densitometric analysis of images was performed using the gel analysis tool available in $\mathrm{Fiji}^{48}$.

\section{References}

1. Gordon DE, Jang GM, Bouhaddou M, et al. A SARS-CoV-2 protein interaction map reveals targets for drug repurposing. Nature. 2020;583(7816):459-468. doi:10.1038/s41586-020-2286-9

2. Riva L, Yuan S, Yin X, et al. Discovery of SARS-CoV-2 antiviral drugs through large-scale compound repurposing. Nature. July 2020:1-11. doi:10.1038/s41586-020-2577-1

3. Choudhary S, Malik YS, Tomar S. Identification of SARS-CoV-2 Cell Entry Inhibitors by Drug Repurposing Using in silico Structure-Based Virtual Screening Approach. Front Immunol. 2020;11:1664. doi:10.3389/fimmu.2020.01664

4. Bouhaddou M, Memon D, Meyer B, et al. The Global Phosphorylation Landscape of SARS-CoV-2 Infection. Cell. 2020;182(3):685-712.e19. doi:10.1016/j.cell.2020.06.034

5. Beigel JH, Tomashek KM, Dodd LE, et al. Remdesivir for the Treatment of Covid-19 Final Report. N Engl J Med. 2020;383(19):1813-1826. doi:10.1056/nejmoa2007764 
6. Biswal M, Diggs S, Xu D, et al. Two conserved oligomer interfaces of NSP7 and NSP8 underpin the dynamic assembly of SARS-CoV-2 RdRP. Nucleic Acids Res. 2021;49(10):5956-5966. doi:10.1093/nar/gkab370

7. Sokol RJ, Heubi JE, Butler-Simon N, McClung HJ, Lilly JR, Silverman A. Treatment of vitamin E deficiency during chronic childhood cholestasis with oral d-a-tocopheryl polyethylene glycol-1000 succinate. Gastroenterology. 1987;93(5):975-985. doi:10.1016/0016-5085(87)90559-2

8. Sokol RJ, Butler-Simon N, Conner C, et al. Multicenter trial of d- $\alpha$-tocopheryl polyethylene glycol 1000 succinate for treatment of vitamin E deficiency in children with chronic cholestasis. Gastroenterology. 1993;104(6):1727-1735. doi:10.1016/00165085(93)90652-S

9. Varma MVS, Panchagnula R. Enhanced oral paclitaxel absorption with vitamin E-TPGS: Effect on solubility and permeability in vitro, in situ and in vivo. Eur J Pharm Sci. 2005;25(4-5):445-453. doi:10.1016/j.ejps.2005.04.003

10. Drosten C, Günther S, Preiser W, et al. Identification of a Novel Coronavirus in Patients with Severe Acute Respiratory Syndrome. N Engl J Med. 2003;348(20):1967-1976. doi:10.1056/nejmoa030747

11. Zaki AM, van Boheemen S, Bestebroer TM, Osterhaus ADME, Fouchier RAM. Isolation of a Novel Coronavirus from a Man with Pneumonia in Saudi Arabia. N Engl $\mathrm{J}$ Med. 2012;367(19):1814-1820. doi:10.1056/nejmoa1211721

12. Zhu N, Zhang D, Wang W, et al. A Novel Coronavirus from Patients with Pneumonia in China, 2019. N Engl J Med. 2020;382(8):727-733. doi:10.1056/nejmoa2001017

13. Andreano E, Rappuoli R. SARS-CoV-2 escaped natural immunity, raising questions 
about vaccines and therapies. Nat Med. 2021;27(5):759-761. doi:10.1038/s41591-02101347-0

14. Cele S, Gazy I, Jackson L, et al. Escape of SARS-CoV-2 501Y.V2 from neutralization by convalescent plasma. Nature. 2021;593(7857):142-146. doi:10.1038/s41586-021-03471w

15. Chen RE, Zhang X, Case JB, et al. Resistance of SARS-CoV-2 variants to neutralization by monoclonal and serum-derived polyclonal antibodies. Nat Med. 2021;27(4):717-726. doi:10.1038/s41591-021-01294-w

16. Kemp SA, Collier DA, Datir RP, et al. SARS-CoV-2 evolution during treatment of chronic infection. Nature. 2021;592(7853):277-282. doi:10.1038/s41586-021-03291-y

17. McCarthy KR, Rennick LJ, Nambulli S, et al. Recurrent deletions in the SARS-CoV-2 spike glycoprotein drive antibody escape. Science (80- ). 2021;371(6534):1139-1142. doi:10.1126/science.abf6950

18. Seow J, Graham C, Merrick B, et al. Longitudinal observation and decline of neutralizing antibody responses in the three months following SARS-CoV-2 infection in humans. Nat Microbiol. 2020;5(12):1598-1607. doi:10.1038/s41564-020-00813-8

19. Siddiqi HK, Mehra MR. COVID-19 illness in native and immunosuppressed states: A clinical-therapeutic staging proposal. J Hear Lung Transplant. 2020;39(5):405-407. doi:10.1016/j.healun.2020.03.012

20. Hansen $\mathrm{CH}$, Michlmayr D, Gubbels SM, Mølbak K, Ethelberg S. Assessment of protection against reinfection with SARS-CoV-2 among 4 million PCR-tested individuals in Denmark in 2020: a population-level observational study. Lancet. 2021;397(10280):1204-1212. doi:10.1016/S0140-6736(21)00575-4 
21. Ratzan S, Schneider EC, Hatch H, Cacchione J. Missing the Point - How Primary Care Can Overcome Covid-19 Vaccine "Hesitancy." N Engl J Med. May 2021. doi:10.1056/nejmp2106137

22. Beyrer C, Allotey P, Amon JJ, et al. Human rights and fair access to COVID-19 vaccines: the International AIDS Society-Lancet Commission on Health and Human Rights. Lancet. 2021;397(10284):1524-1527. doi:10.1016/S0140-6736(21)00708-X

23. Williams TC, Burgers WA. SARS-CoV-2 evolution and vaccines: cause for concern? Lancet Respir Med. 2021;9(4):333-335. doi:10.1016/S2213-2600(21)00075-8

24. Loewe S, Muischnek H. Über Kombinationswirkungen - Mitteilung: Hilfsmittel der Fragestellung. Arch für Exp Pathol und Pharmakologie. 1926;114(5-6):313-326. doi:10.1007/BF01952257

25. Foucquier J, Guedj M. Analysis of drug combinations: current methodological landscape. Pharmacol Res Perspect. 2015;3(3):149. doi:10.1002/prp2.149

26. Dintaman JM, Silverman JA. Inhibition of P-glycoprotein by D-a-tocopheryl polyethylene glycol 1000 succinate (TPGS). Pharm Res. 1999;16(10):1550-1556.

doi:10.1023/A:1015000503629

27. Collnot EM, Baldes C, Schaefer UF, Edgar KJ, Wempe MF, Lehr CM. Vitamin e TPGS Pglycoprotein inhibition mechanism: Influence on conformational flexibility, intracellular ATP levels, and role of time and site of access. Mol Pharm. 2010;7(3):642-651. doi:10.1021/mp900191s

28. Yin W, Mao C, Luan X, et al. Structural basis for inhibition of the RNA-dependent RNA polymerase from SARS-CoV-2 by remdesivir. Science (80- ). 2020;368(6498):1499-1504. doi:10.1126/science.abc1560 
29. Gordon CJ, Tchesnokov EP, Woolner E, et al. Remdesivir is a direct-acting antiviral that inhibits RNA-dependent RNA polymerase from severe acute respiratory syndrome coronavirus 2 with high potency. J Biol Chem. 2020;295(20):6785-6797. doi:10.1074/jbc.RA120.013679

30. Puig-Rigall J, Grillo I, Dreiss CA, González-Gaitano G. Structural and Spectroscopic Characterization of TPGS Micelles: Disruptive Role of Cyclodextrins and Kinetic Pathways. 2017. doi:10.1021/ACS.LANGMUIR.7B00701

31. Walker AP, Fan H, Keown JR, Grimes JM, Fodor E. Identification of guanylyltransferase activity in the SARS-CoV-2 RNA polymerase. bioRxiv. 2021.

32. Jensen SK, Engberg RM, Hedemann MS. All-rac- $\alpha$-tocopherol acetate is a better vitamin E source than all- rac- $\alpha$-tocopherol succinate for broilers. J Nutr. 1999;129(7):1355-1360. doi:10.1093/jn/129.7.1355

33. Yu L, Bridgers A, Polli J, et al. Vitamin E-TPGS increases absorption flux of an HIV protease inhibitor by enhancing its solubility and permeability. Pharm Res. 1999;16(12):1812-1817. doi:10.1023/A:1018939006780

34. Shin SC, Kim J. Physicochemical characterization of solid dispersion of furosemide with TPGS. Int J Pharm. 2003;251(1-2):79-84. doi:10.1016/S0378-5173(02)00586-0

35. Ismailos G, Reppas C, Macheras P. Enhancement of cyclosporin A solubility by dalphatocopheryl-polyethylene-glycol-1000 succinate (TPGS). Eur J Pharm Sci. 1994;1(5):269-271. doi:10.1016/0928-0987(94)90021-3

36. Yang C, Wu T, Qi Y, Zhang Z. Recent advances in the application of vitamin E TPGS for drug delivery. Theranostics. 2018;8(2):464-485. doi:10.7150/thno.22711

37. Sadoqi M, Lau-Cam CA, Wu SH. Investigation of the micellar properties of the tocopheryl 
polyethylene glycol succinate surfactants TPGS 400 and TPGS 1000 by steady state fluorometry. J Colloid Interface Sci. 2009;333(2):585-589. doi:10.1016/j.jcis.2009.01.048

38. Collnot EM, Baldes C, Wempe MF, et al. Influence of vitamin E TPGS poly(ethylene glycol) chain length on apical efflux transporters in Caco-2 cell monolayers. J Control Release. 2006;111(1-2):35-40. doi:10.1016/j.jconrel.2005.11.005

39. Hosomi A, Arita M, Sato Y, et al. Affinity for A-Tocopherol Transfer Protein as a Determinant of the Biological Activities of Vitamin E Analogs. Vol 409. Elsevier B.V.; 1997. doi:10.1016/S0014-5793(97)00499-7

40. Brigelius-Flohé R, Traber MG. Vitamin E: function and metabolism. FASEB J. 1999;13(10):1145-1155. doi:10.1096/fasebj.13.10.1145

41. Traber MG. Determinants of plasma vitamin E concentrations. Free Radic Biol Med. 1994;16(2):229-239. doi:10.1016/0891-5849(94)90148-1

42. Tchesnokov EP, Feng JY, Porter DP, Götte M. Mechanism of inhibition of ebola virus RNA-dependent RNA polymerase by remdesivir. Viruses. 2019;11(4):326. doi:10.3390/v11040326

43. Nakagawa K, Lokugamage KG, Makino S. Viral and Cellular mRNA Translation in Coronavirus-Infected Cells. In: Advances in Virus Research. Vol 96. Academic Press Inc.; 2016:165-192. doi:10.1016/bs.aivir.2016.08.001

44. Wang M, Cao R, Zhang L, et al. Remdesivir and chloroquine effectively inhibit the recently emerged novel coronavirus (2019-nCoV) in vitro. Cell Res. 2020;30(3):269-271. doi:10.1038/s41422-020-0282-0

45. Walker AP, Fan H, Keown JR, Grimes JM, Fodor E. Identification of guanylyltransferase activity in the SARS-CoV-2 RNA polymerase. bioRxiv. 2021. doi:2021.03.17.435913 
46. Kokic G, Hillen HS, Tegunov D, et al. Mechanism of SARS-CoV-2 polymerase stalling by remdesivir. Nat Commun. 2021;12(1):1-7. doi:10.1038/s41467-020-20542-0

47. Friedman DP, Byrd WE, Kiselyov O, Hemann J. The Reasoned Schemer. 2nd ed. Cambridge, MA, USA: MIT Press; 2018.

48. Schindelin J, Arganda-Carreras I, Frise E, et al. Fiji: an open-source platform for biological-image analysis. Nat Methods 2012 97. 2012;9(7):676-682.

doi:10.1038/nmeth.2019

49. R Core Team. R: A language and environment for statistical computing. 2020. https://www.r-project.org/.

50. RStudio Team. RStudio: Integrated Development for R. 2020. http://www.rstudio.com/.

51. Wickham H, Averick M, Bryan J, et al. Welcome to the Tidyverse. J Open Source Softw. 2019;4(43):1686. doi:10.21105/joss.01686

52. Sievert C. Interactive web-based data visualization with R, plotly, and shiny. 2020. https://plotly-r.com.

53. Ritz C, Baty F, Streibig JC, Gerhard D. Dose-Response Analysis Using R. PLoS One. 2015;10(12):e0146021. doi:10.1371/journal.pone.0146021

54. Young MD, Wakefield MJ, Smyth GK, Oshlack A. Gene ontology analysis for RNA-seq: accounting for selection bias. Genome Biol. 2010;11(2):14. doi:10.1186/gb-2010-11-2-r14

55. Zhao L, Au JLS, Wientjes MG. Comparison of methods for evaluating drug-drug interaction. Front Biosci - Elit. 2010;2 E(1):241-249. doi:10.2741/e86

56. Greco WR, Bravo G, Parsons JC. The search for synergy: a critical review from a response surface perspective. Pharmacol Rev. 1995;47(2). 
57. Straetemans R, O'brien T, Wouters L, et al. Design and Analysis of Drug Combination Experiments. Biometrical J. 2005;47(3):299-308. doi:10.1002/bimj.200410124

58. Grabovsky Y, Tallarida RJ. Isobolographic analysis for combinations of a full and partial agonist: Curved isoboles. J Pharmacol Exp Ther. 2004;310(3):981-986. doi:10.1124/jpet.104.067264

59. Dobin A, Davis CA, Schlesinger F, et al. STAR: Ultrafast universal RNA-seq aligner. Bioinformatics. 2013;29(1):15-21. doi:10.1093/bioinformatics/bts635

60. Anders S, Pyl PT, Huber W. HTSeq-A Python framework to work with high-throughput sequencing data. Bioinformatics. 2015;31(2):166-169. doi:10.1093/bioinformatics/btu638

61. Trott O, Olson AJ. AutoDock Vina: Improving the speed and accuracy of docking with a new scoring function, efficient optimization, and multithreading. J Comput Chem. 2009;31(2):NA-NA. doi:10.1002/jcc.21334

62. Gao Y, Yan L, Huang Y, et al. Structure of the RNA-dependent RNA polymerase from COVID-19 virus. Science (80- ). 2020;368(6492):779-782. doi:10.1126/science.abb7498 


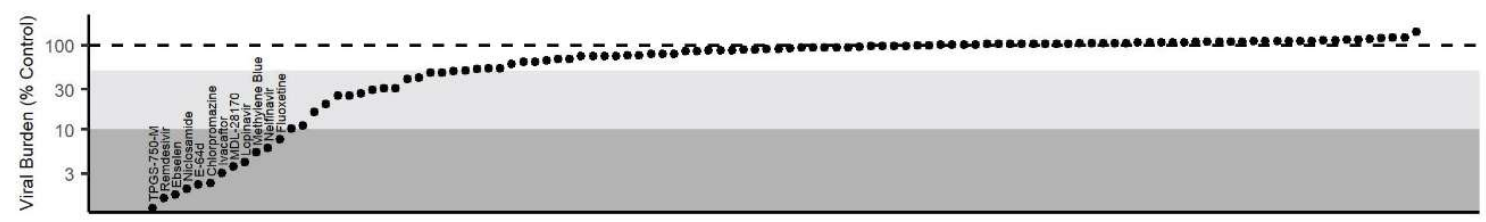

B
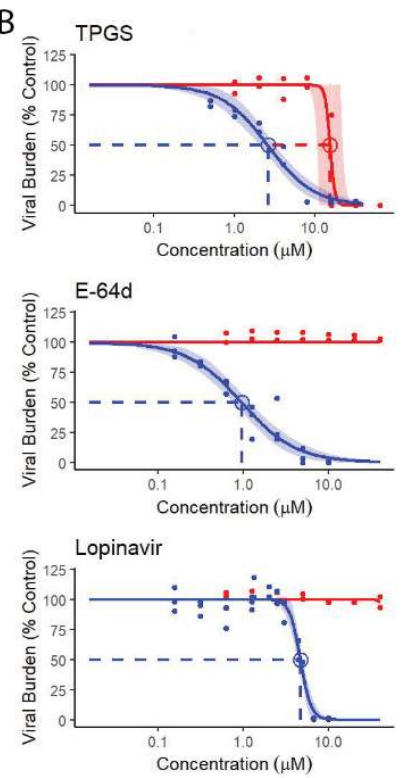

C
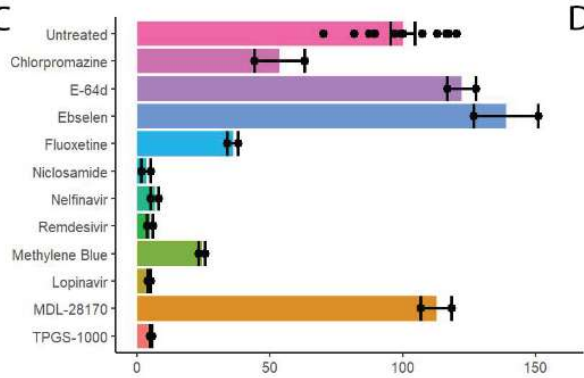

Cells
Treatment
Virus

SARS-CoV-2
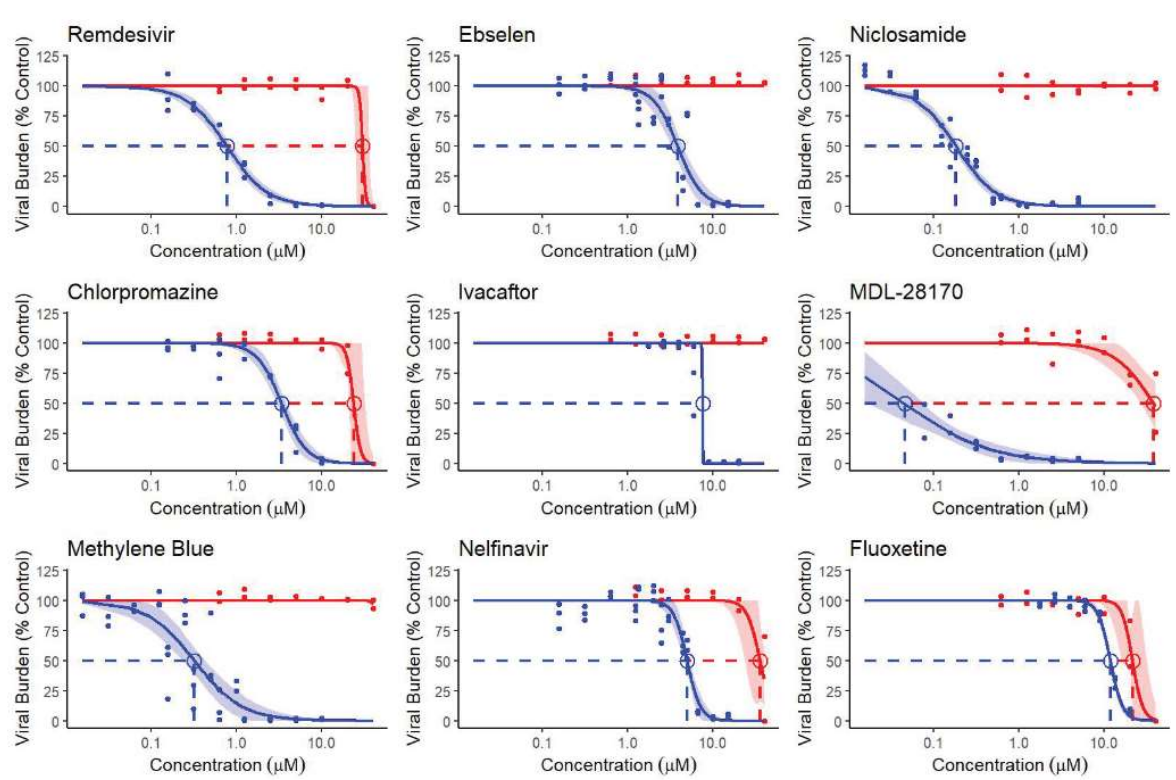

E
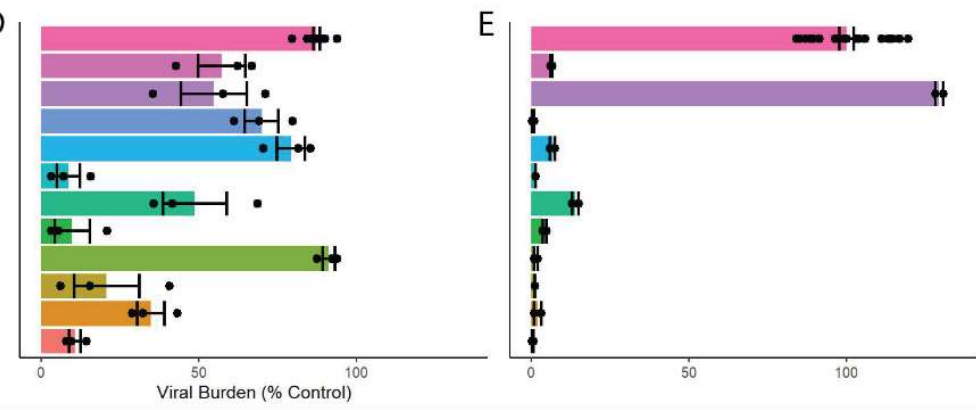

VeroE6

VeroE6

$24 \mathrm{hpi}$

SARS-COV-2
0 hpi

oc43

Figure 1. Identification and characterization of twelve FDA-approved compounds with antiviral activity against SARS-CoV-2. A) A graph depicting rank-ordered antiviral activity against SARS-CoV-2 at $10 \mu \mathrm{M}$ for all drugs tested. Points represent the mean value from two independent experiments. B) Dose response curves for antiviral activity (blue) and viability (red) in VeroE6 cells. The shaded region around each curve represents the 95\% confidence interval, while dots represent technical replicates pooled from two independent experiments. C-E) Antiviral activity of the top drugs at $10 \mu \mathrm{M}$ in C) SARS-CoV-2-infected Calu3 cells, D) SARSCoV-2-infected VeroE6 cells treated from $24 \mathrm{hpi}$ until $48 \mathrm{hpi}$, and E) OC43-infected VeroE6 cells. Dots represent technical replicates from one experiment, bars and error bars represent the mean plus or minus the standard error of the mean. All experiments performed at least twice. 

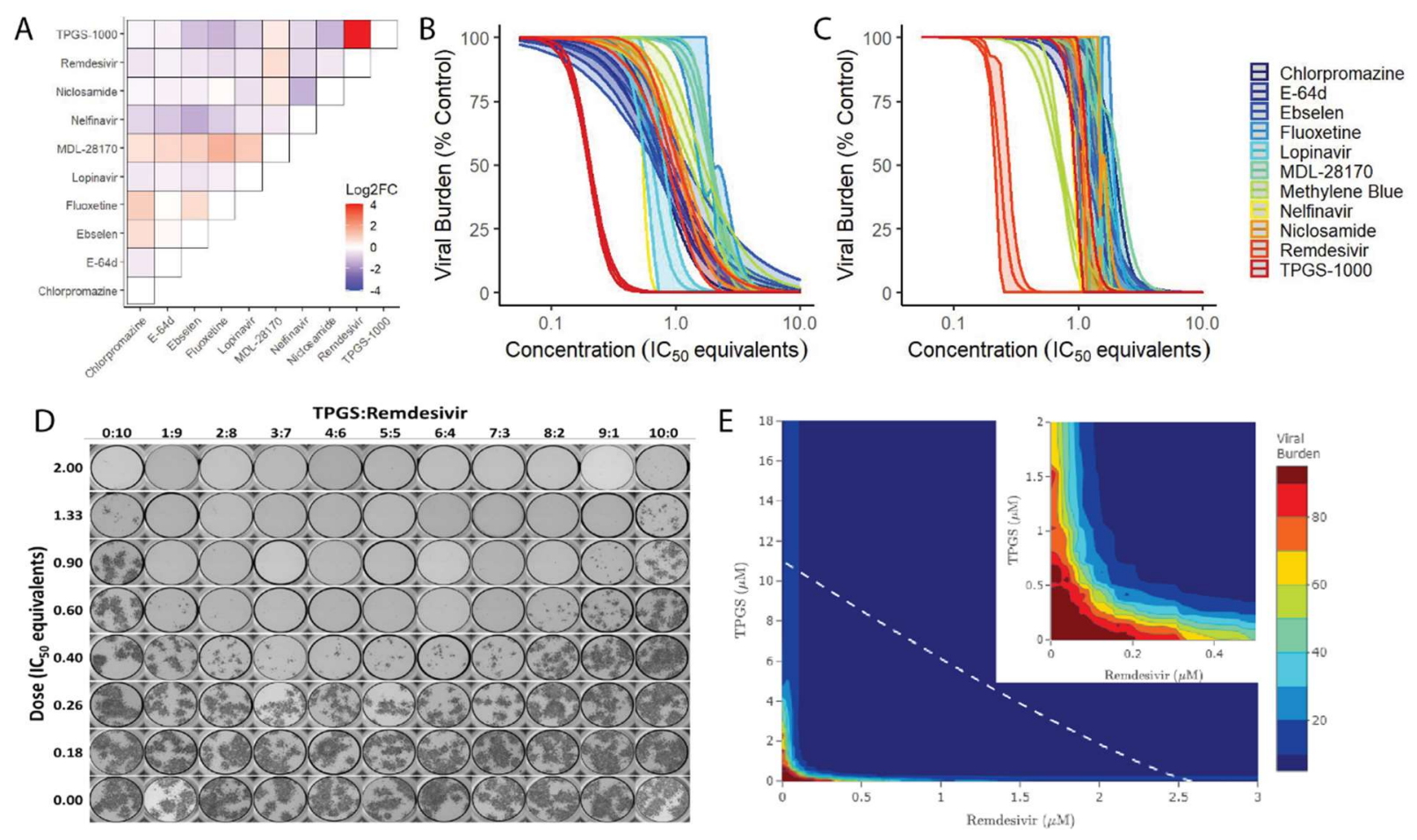

Figure 2. TPGS synergizes with remdesivir. A) Heatmap of the log2 fold change in potency for indicated combinations of the top results from screening. Values determined from three technical replicates. B,C) Curve-shift analysis for top drugs combined with B) Remdesivir or C) TPGS. Shaded regions surrounding each curve represent the $95 \%$ confidence interval for the doseresponse model. D) Representative whole-well images captured for E) response-surface analysis of combinations of remdesivir and TPGS. The white, dashed line represents the predicted isobole for the $\mathrm{IC}_{90}$ while the inset magnifies the origin of the graph. Two technical replicates were used to determine the antiviral activity at each point assayed. All experiments were performed at least twice. 

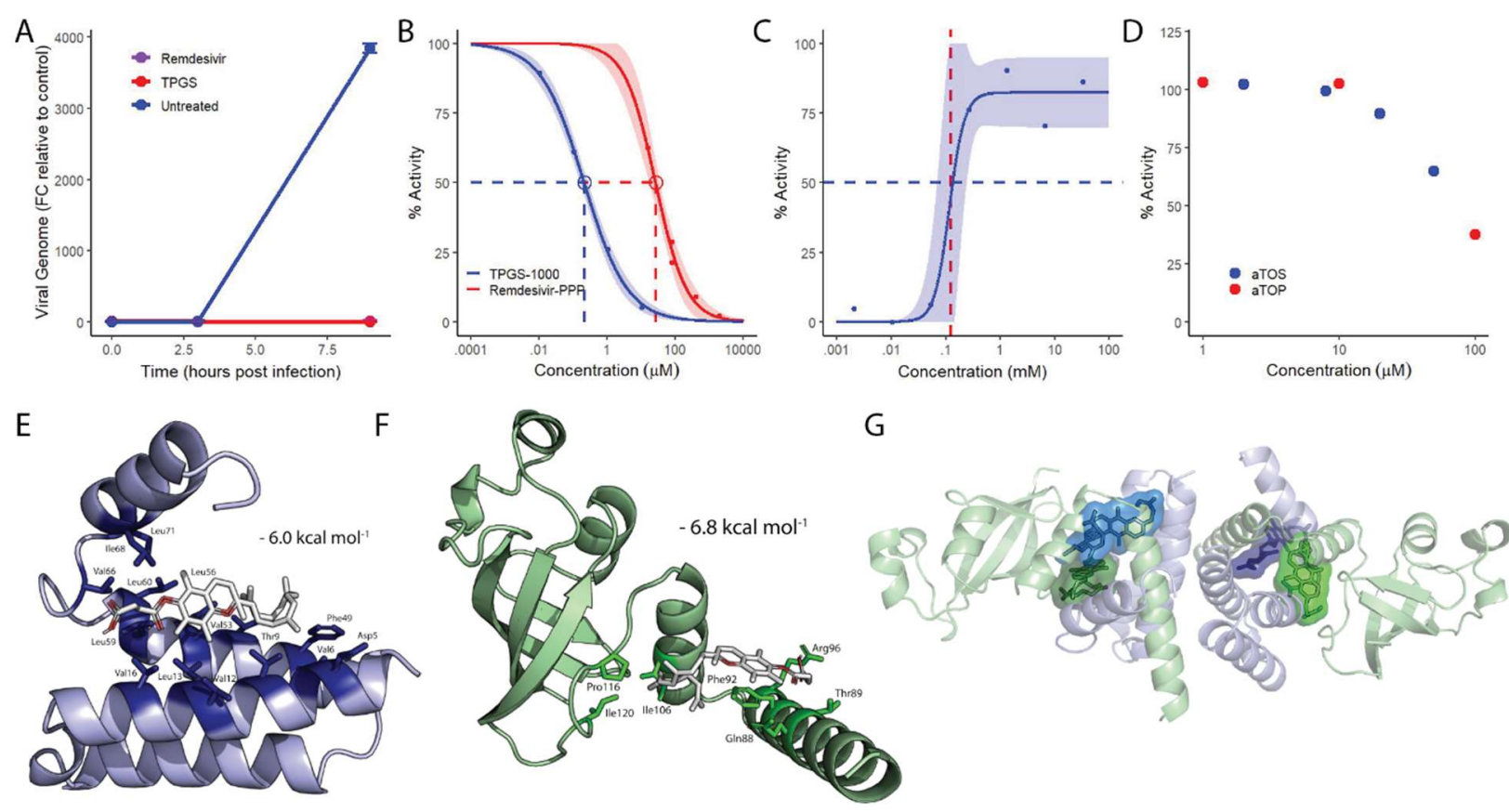

G

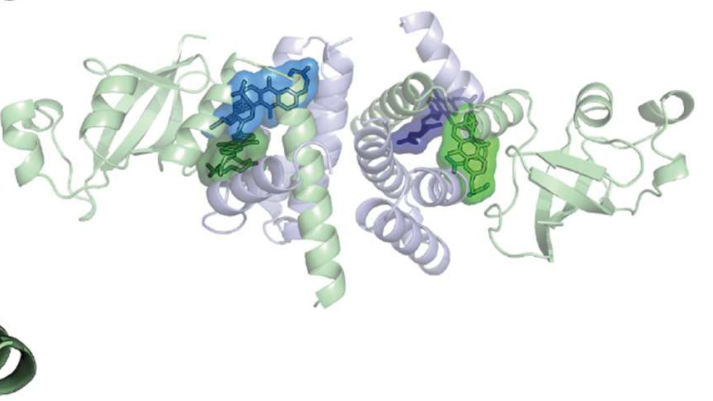

Figure 3. Water-soluble tocopherols inhibit the transcriptional activity of SARS-CoV-2 RNA-dependent RNA polymerase. A) Graph representing the fold change of SARS-CoV-2 genome in infected VeroE6 cells relative to initial uptake in the untreated control. Remdesivir and TPGS were given at a fully effective dose, $10 \mu \mathrm{M}$ and $30 \mu \mathrm{M}$, respectively. Points and error bars represent the mean +/- SEM calculated from two technical replicates. B) Dose-response curves for the transcriptional activity of the SARS-CoV-2 replication complex treated with TPGS (blue) and remdesivir (red). The shaded region represents the $95 \% \mathrm{Cl}$ and points represent replicates from a single experiment. C) Dose-response curve for the transcriptional activity of the SARSCoV-2 replication complex treated with high concentrations of TPGS, with $50 \%$ activity (blue, dashed line) and the critical micelle concentration of TPGS (red, dashed line) indicated. The upper limit of the model was not fixed. Points represent replicates from a single experiment. D) Doseresponse plot for increasing concentrations of aTOS (blue) and aTOP (red). Points represent replicates from a single experiment. E) Docking model of the most favorable pose for aTOS (white) interacting with conserved, hydrophobic residues (dark blue, named) within NSP7 (light blue). F) Docking model of the most favorable pose for aTOS (white) interacting with conserved, hydrophobic residues (dark green, named) within NSP8 (light green). G) Representation of the heterotetrameric structure of NSP7 (light blue) and NSP8 (light green) with the most favorable poses of aTOS interacting individually with each NSP7 (dark blue) and NSP 8 (dark green) superimposed. 

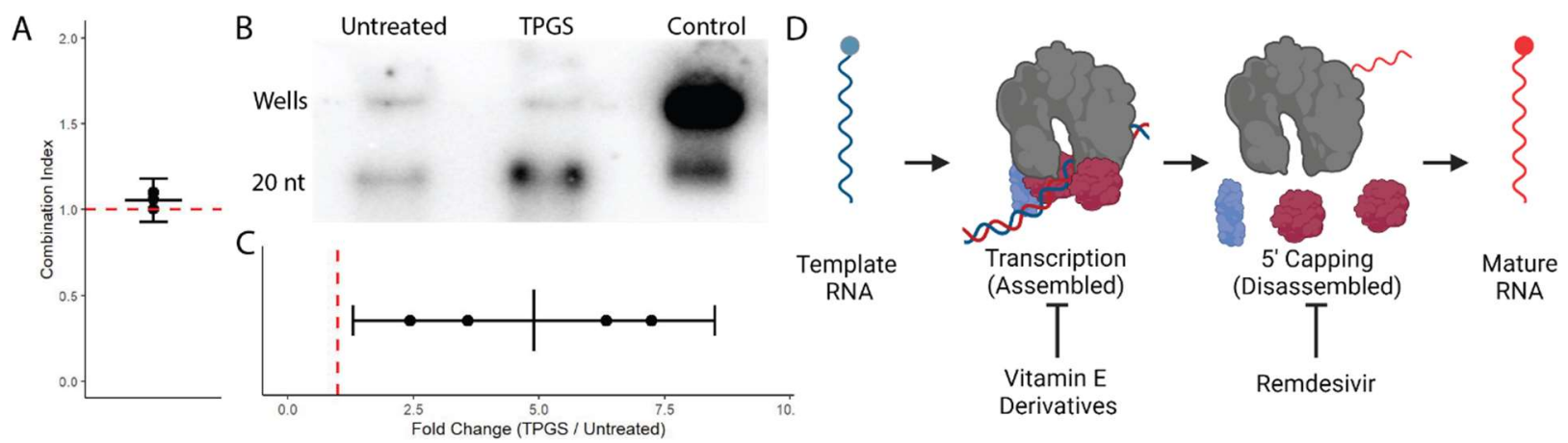

Figure 4. TPGS increases guanylyl transferase activity of SARS-CoV-2 RdRp. A) Plot of the combination index calculated for mixtures of TPGS and remdesivir at variable potency ratios $(0.1$ - 0.7 TPGS:remdesivir) and doses $\left(0.4-1 I_{50}\right.$ equivalents). Each point represents the combination index determined in an independent experiment, the black line and error bars represent the mean $+/$ - the $95 \% \mathrm{Cl}$, and the red, dashed line represents a combination index of 1. B) Representative raw output from guanylyl transferase assay. C) Quantification of results from the guanylyl transferase assay pooling four replicates across three independent experiments. The black line and error bars represent the mean $+/$ - the $95 \% \mathrm{Cl}$ and the red, and the red, dashed line represents a combination index of 1 . D) Depiction of our proposed model for synergy between water-soluble derivatives of $\alpha$-tocopherol and remdesivir. 
A. Selected proteins involved in host-viral interactions \& host response

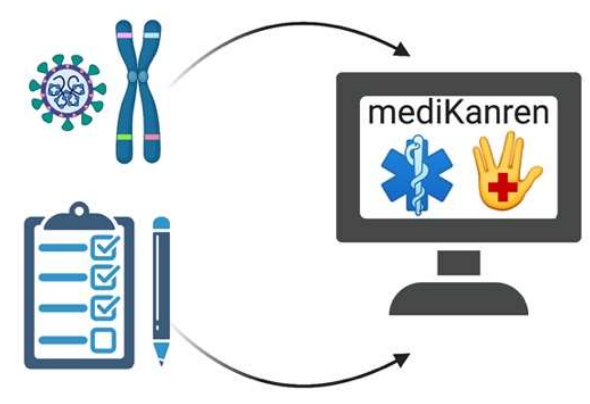

B. Drugs directly \& indirectly regulating host or viral mechanisms

I. Drugs inhibiting viral or host proteins

II. Drugs activating down regulators of viral or host proteins

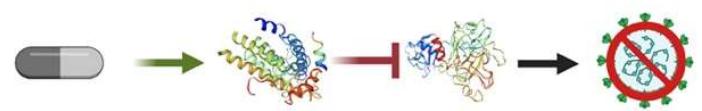

III. Drugs inhibiting up regulators of viral or host proteins

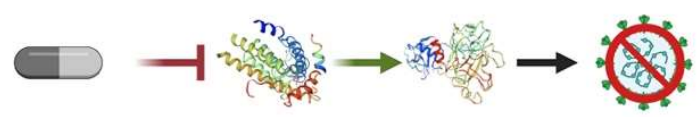

C. Filtration and prioritization drug candidates

\begin{tabular}{|c|c|c|c|c|c|}
\hline \multirow{4}{*}{$\begin{array}{l}\text { Tested } \\
\text { in vitro }\end{array}$} & & $\downarrow$ Virus & $\uparrow$ Virus & $\downarrow$ sympt. & $\uparrow$ sympt. \\
\hline & Priority candidates & $\sqrt{ } \sqrt{ }$ & $X$ & $\sqrt{ } \sqrt{ }$ & $x$ \\
\hline & Secondary candidates & $\sqrt{ } \sqrt{ }$ & X & $?$ & X \\
\hline & Tertiary candidates & $\checkmark$ & $?$ & $?$ & ? \\
\hline & $\begin{array}{l}\text { Candidates expected } \\
\text { To exacerbate illness }\end{array}$ & $?$ & $\checkmark$ & $?$ & \\
\hline
\end{tabular}

Figure S1. Depiction of expedited generation of candidate drugs based on host-pathogen interactions/pathways and prioritized based on therapeutic potential and risk. A) Host and viral proteins essential for viral replication and genes related to patients' symptoms are input to mediKanren. B) Drugs with both direct and indirect impact on the protein's activity are returned. C) Candidates are selected and given higher priority based on their ability to decrease mechanisms favorable to viral replication or those resulting in COVID-19 symptoms (impacting multiple mechanisms is shown as multiple check marks). A lower priority is given to candidates that would activate mechanisms favorable to viral replication or thought to increase COVID-19 symptoms. Question marks indicate ambiguous or lack of results. Candidates that are predicted to primarily increase viral replication or COVID-19 symptoms are flagged for review by the clinical trial committee. These may be included in observational trials to guide best practices for treating COVID-19 patients. 

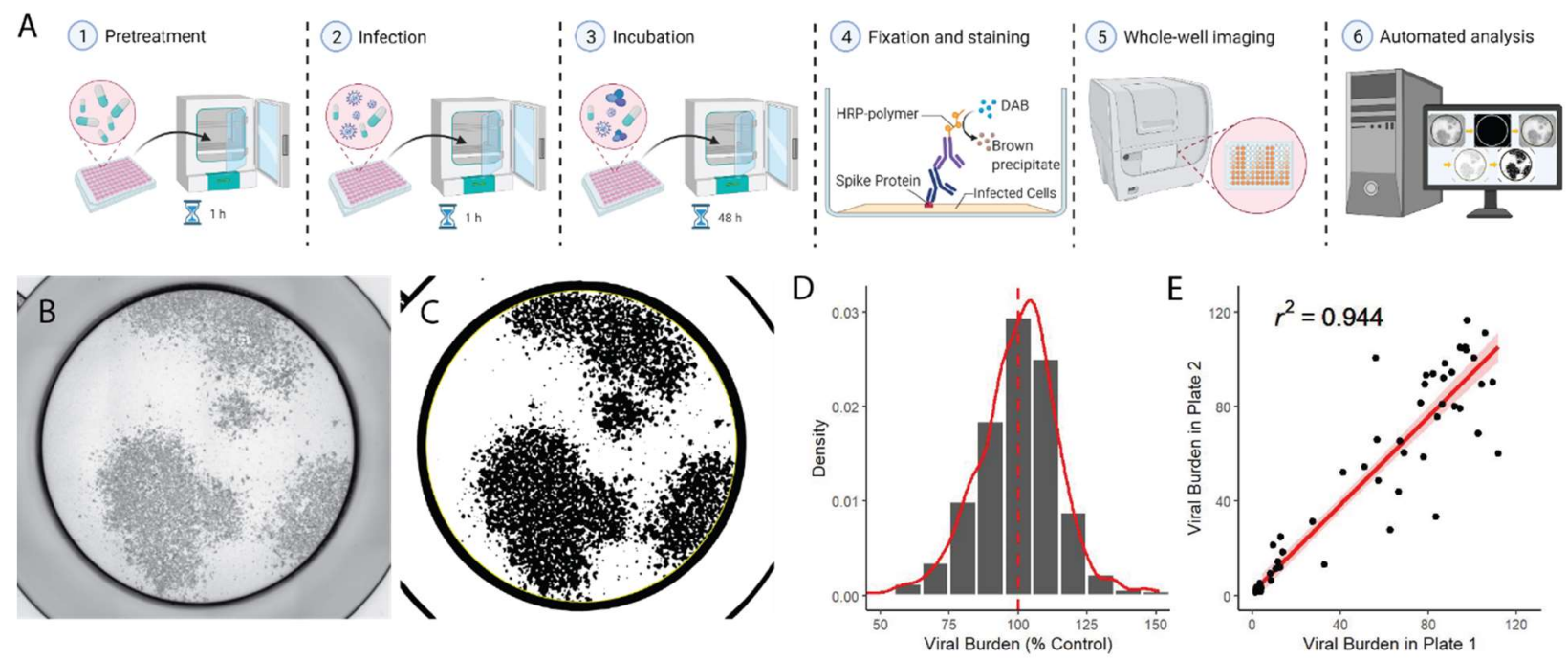

Figure S2. Focus-based antiviral activity assay is automated and reliable. A) An illustration depicting the major steps in the antiviral activity assay used for in vitro screening and follow up experiments. B) Raw and C) processed representative, whole well images from screening-based assay, including the automatically determined well area (yellow border). D) Histogram and density plot (red, solid line) from normalized, untreated control wells $(n=644)$ pooled across all experiments. The mean (red, dashed line) is mathematically centered on 100. E) Scatter plot of individual treatment conditions comparing the viral burden across replicates from two separate plates displaying the value for pearson's correlation coefficient $\left(r^{2}\right)$, the linear regression line (red), and $95 \% \mathrm{Cl}$ (shaded region). 

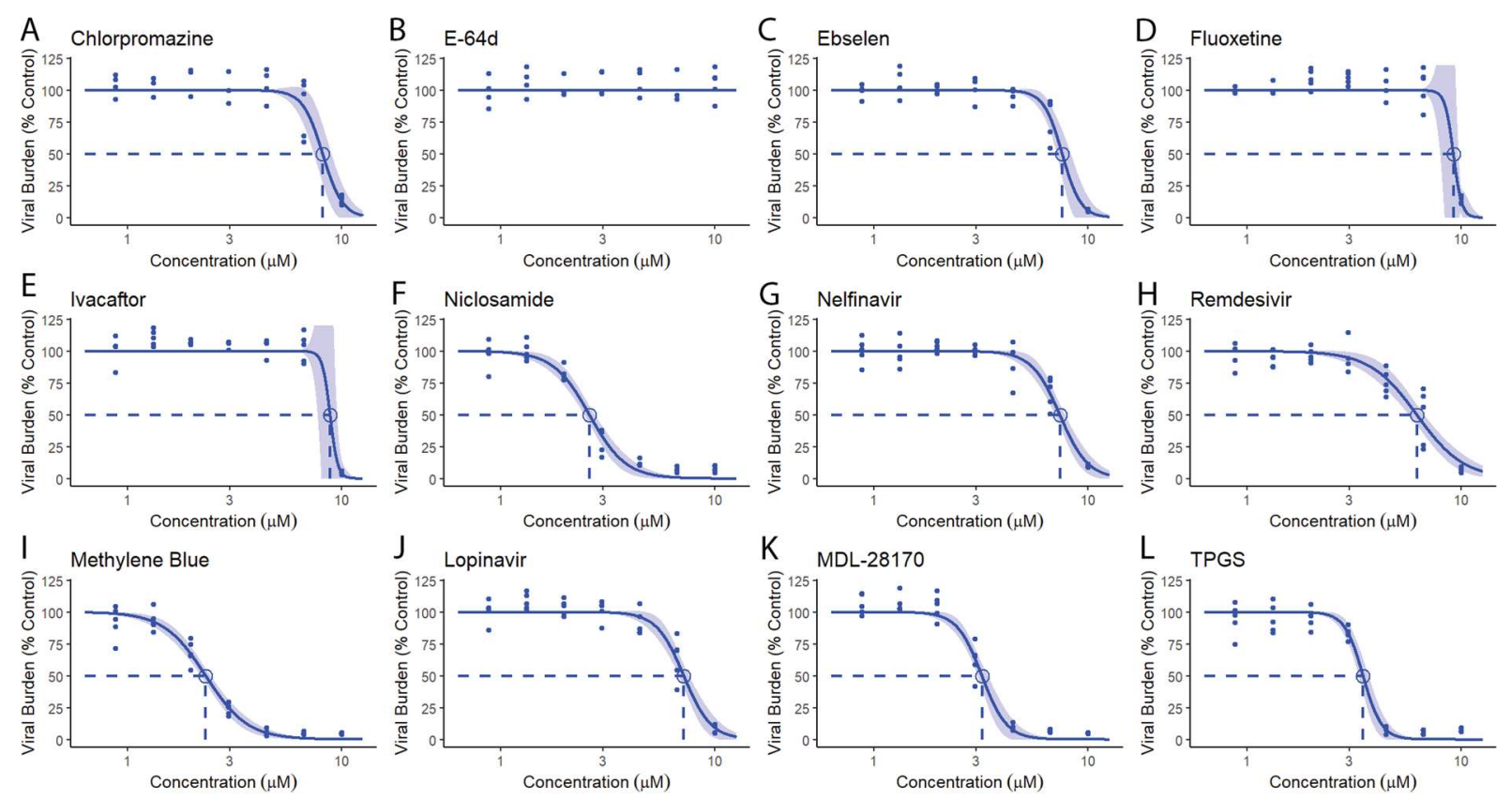

Figure S3. Top compounds inhibit $\beta$-coronavirus OC43. Dose response curves (solid line) and $95 \% \mathrm{Cl}$ (shaded region) for the antiviral activity of the top candidates against OC43. Points represent technical replicates pooled from two independent experiments, and the IC50 is indicated by the dashed lines and circle. 

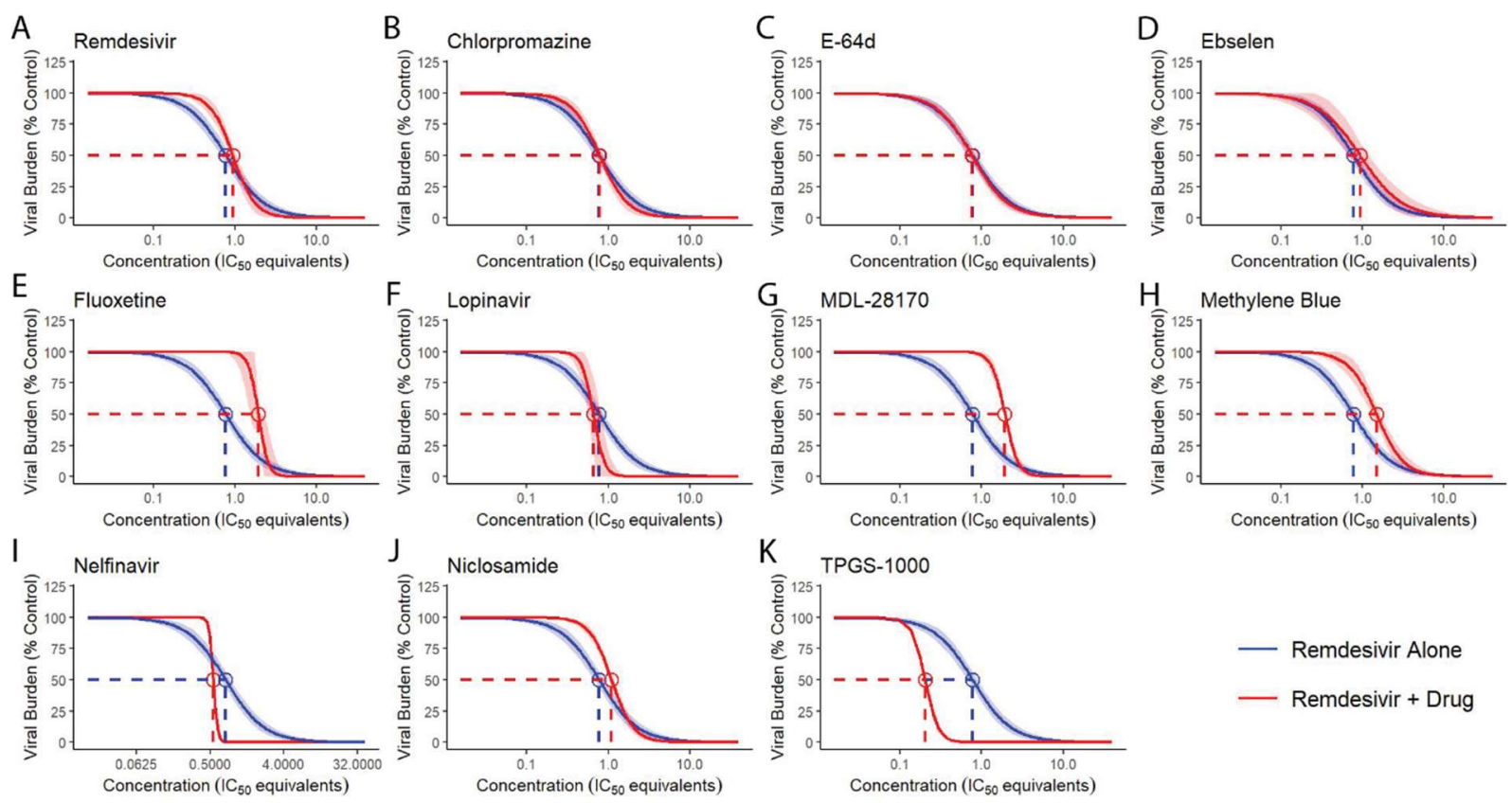

- Remdesivir Alone

— Remdesivir + Drug

Figure S4. Most compounds combine with remdesivir in an additive fashion. Dose response curves (solid line) and $95 \% \mathrm{Cl}$ (shaded region) for remdesivir alone (blue) and remdesivir in an equipotent mixture with the indicated drug (red). 

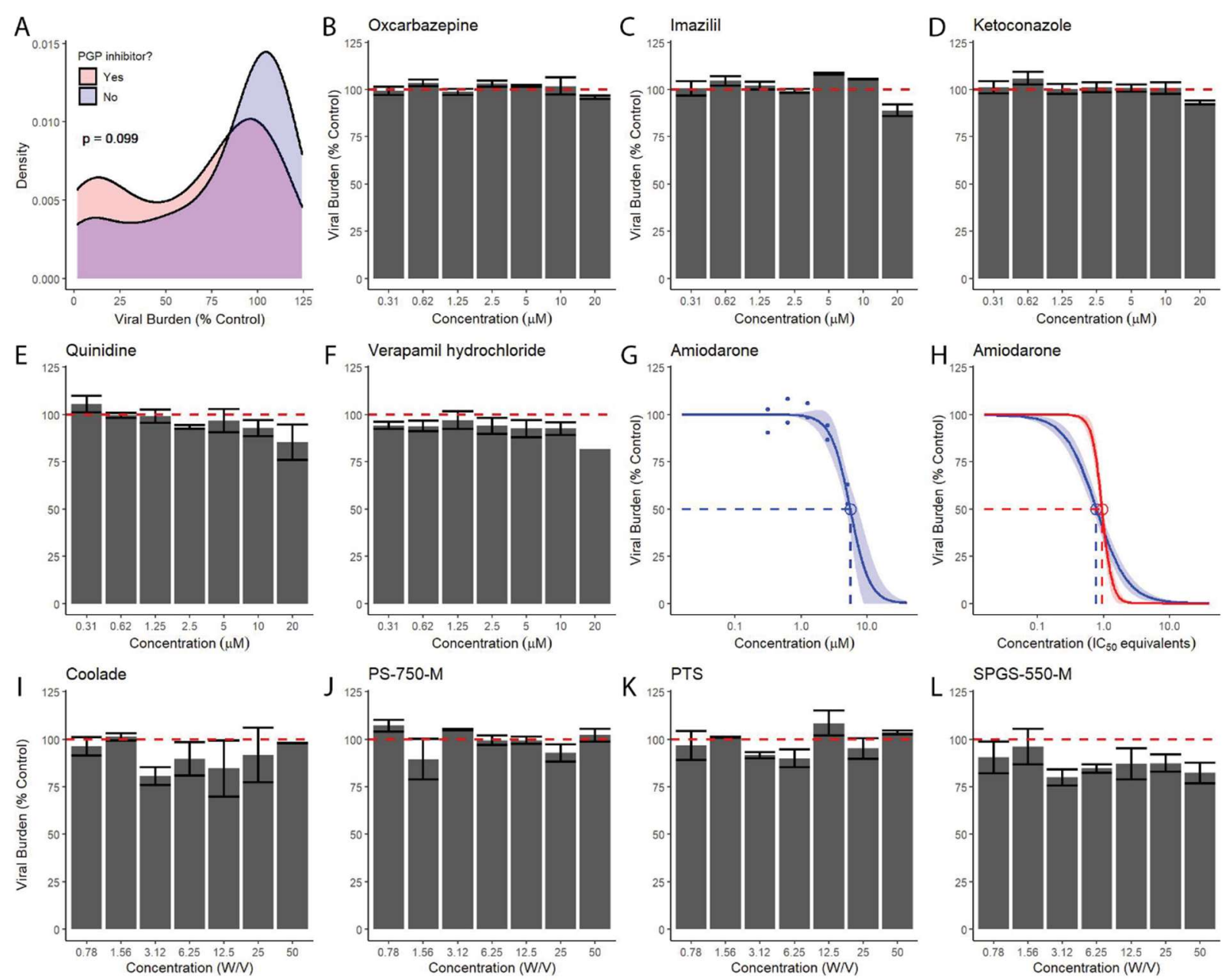

Figure S5. PGP-inhibition and surfactant activity do not account for the antiviral activity of TPGS. A) Density plots for screened drugs classified by DrugBank as inhibitors of PGP (red; $n=$ 34) or not (blue; $n=63$ ). Statistical significance determined by Kolmogorov-Smirnov test. B-F) Column graphs representing the mean +/- SEM for B) an inducer or C-F) inhibitors of PGP activity, determined by two technical replicates in a single experiment. G-H) Dose response curves (solid) and $95 \% \mathrm{Cl}$ (shaded) for $\mathrm{G}$ ) Amiodarone, $\mathrm{H}$ ) amiodarone plus remdesivir (red), or remdesivir alone (blue). Dots in $\mathrm{G}$ ) represent individual replicates. I-L) Column graphs representing the mean +/- SEM determined by two technical replicates in a single experiment for surfactants with structural similarity to TPGS. A minimum of two independent experiments were performed for all panels. 

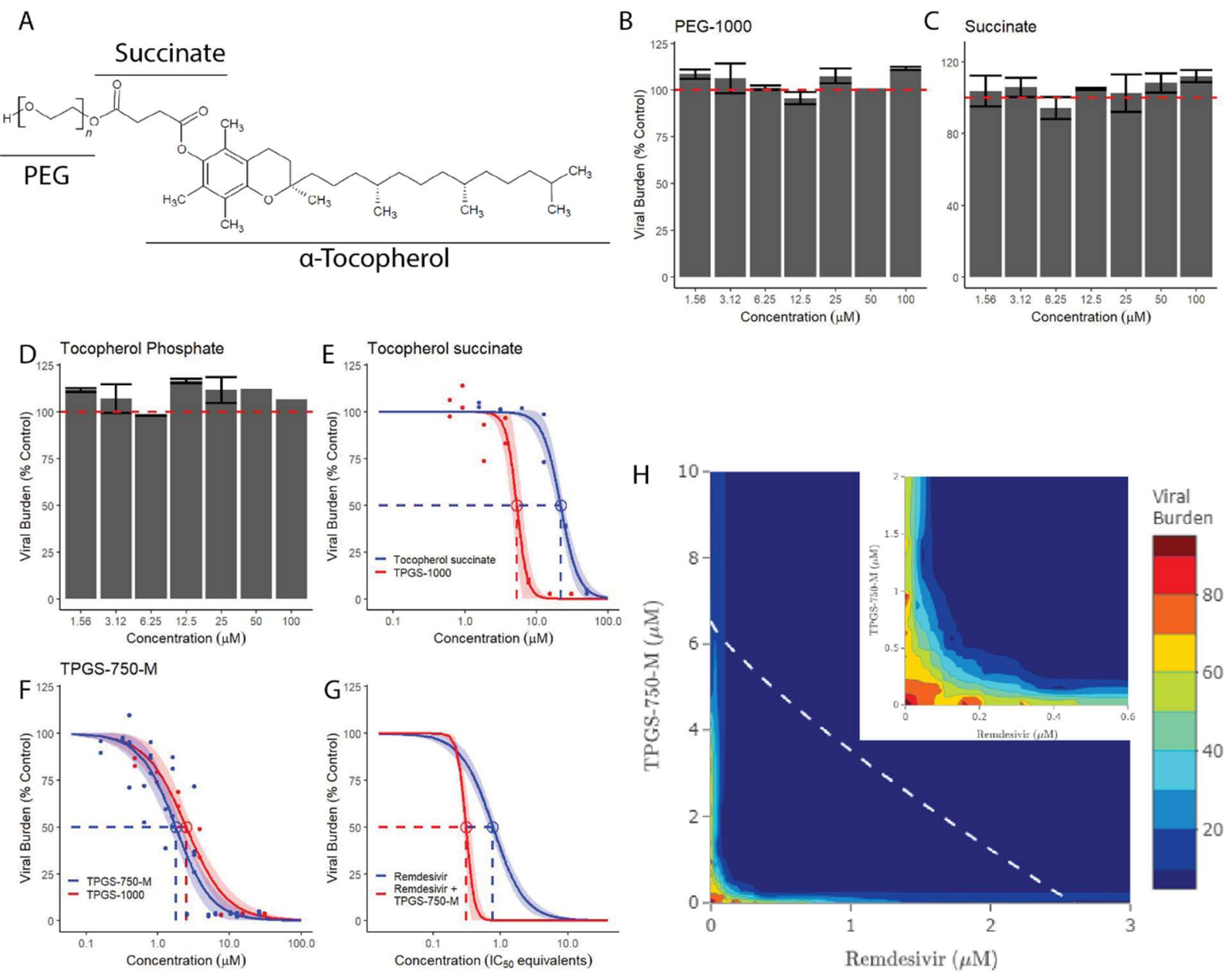

Figure S6. Water-soluble derivatives of Vitamin E inhibit SARS-CoV-2 replication. A) Molecular structure of a generic TPGS molecule. For $\alpha$ TOS, $n=0$, for TPGS, $n=\sim 22$. B-D) Column graphs representing the mean +/- SEM determined by two technical replicates in a single experiment for constituents of TPGS. E-G) Dose response curves (solid) and 95\% $\mathrm{Cl}$ (shaded) for E) aTOS (blue) and TPGS (red); F) TPGS-750-M (blue) and TPGS (red); and G) TPGS-750$M$ plus remdesivir (red) and remdesivir alone (blue). Dots in E,F) represent individual replicates. H) Response-surface analysis for TPGS-750-M in combination with remdesivir. The white, dashed line represents the predicted isobole for the $\mathrm{IC}_{90}$ while the inset magnifies the origin of the graph. Three technical replicates were used to determine the antiviral activity at each point assayed. A minimum of two independent experiments were performed for each panel. 
A
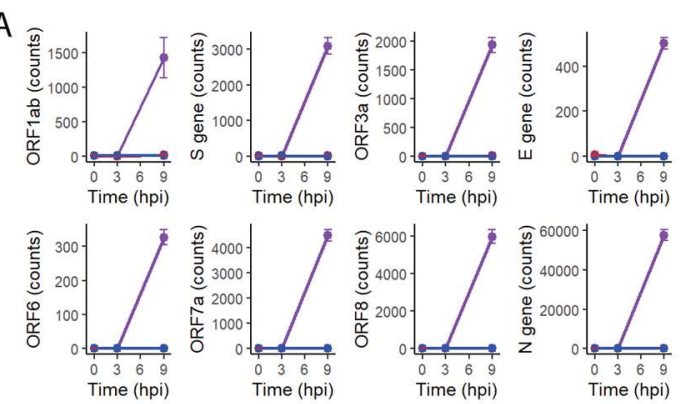

B
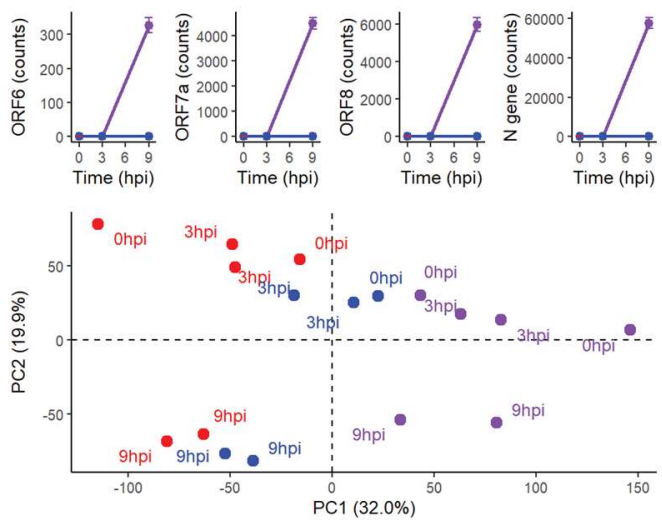

C

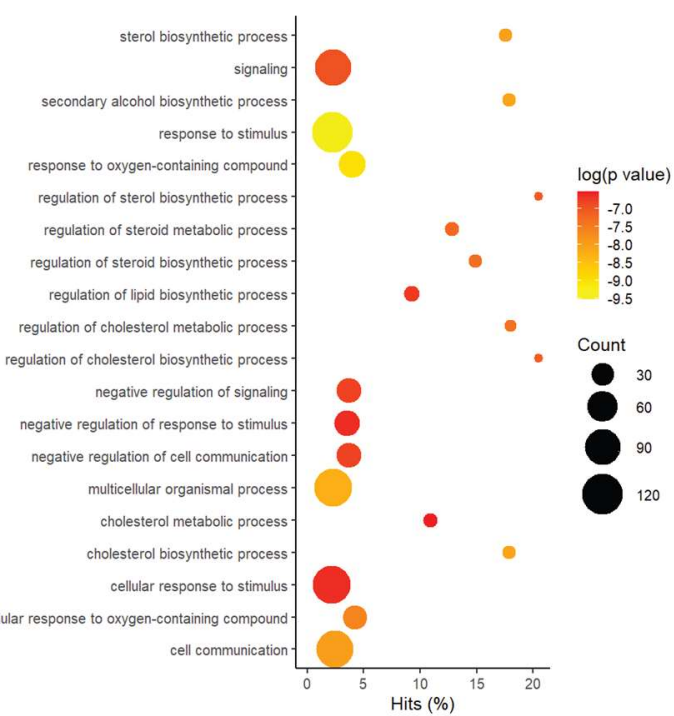

Figure S7. TPGS treatment reshapes the transcriptome of infected host cells. A) Graphs representing SARS-Cov-2 gene expression in infected VeroE6 cells. Remdesivir and TPGS were given at a fully effective dose, $10 \mu \mathrm{M}$ and $30 \mu \mathrm{M}$, respectively. Points and error bars represent the mean +/- SEM calculated from two technical replicates. Group legend is in B). B) Principle component analysis of VeroE6 gene expression from the same samples depicted in (A). C) Analysis of the representation of genes with a $\log _{2}$ fold increase greater than 1.5 in TPGS-treated samples at $9 \mathrm{hpi}$ compared to untreated samples at $9 \mathrm{hpi}$ among gene ontology categories. 


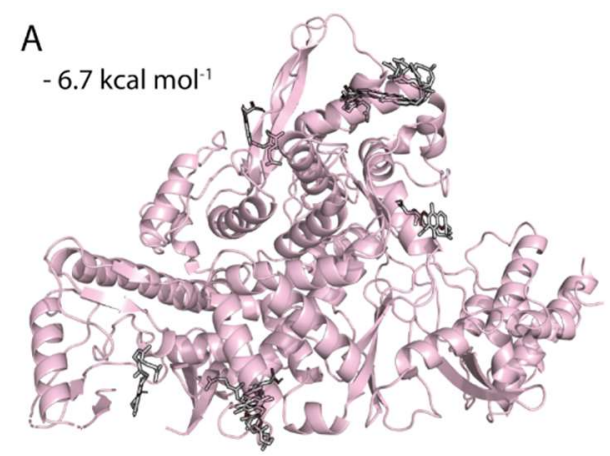

D

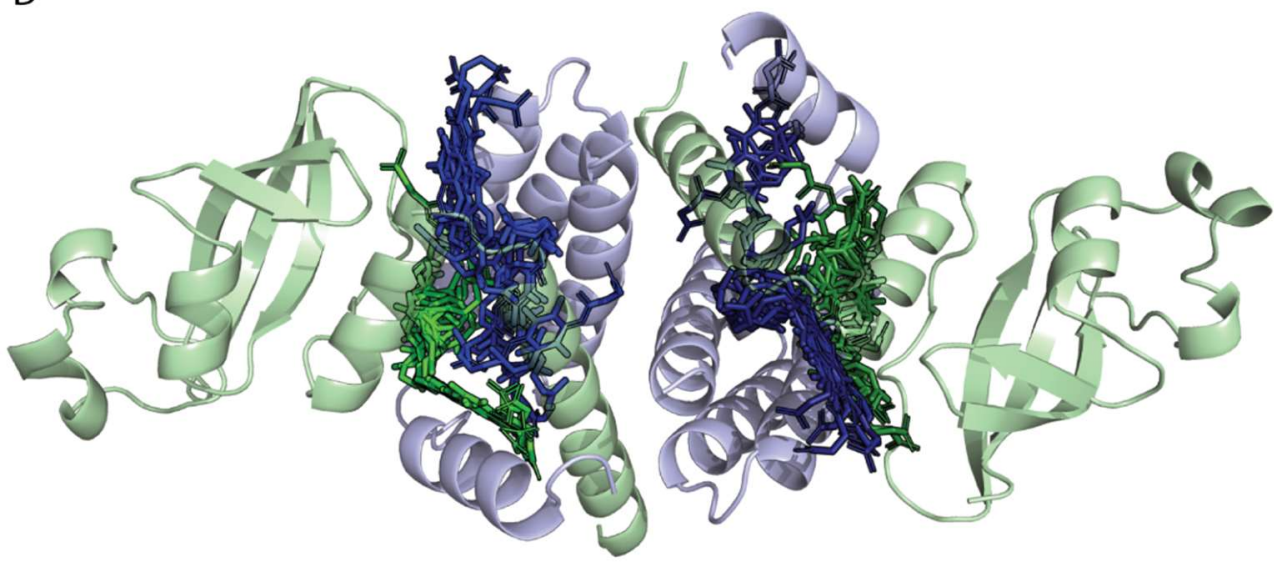

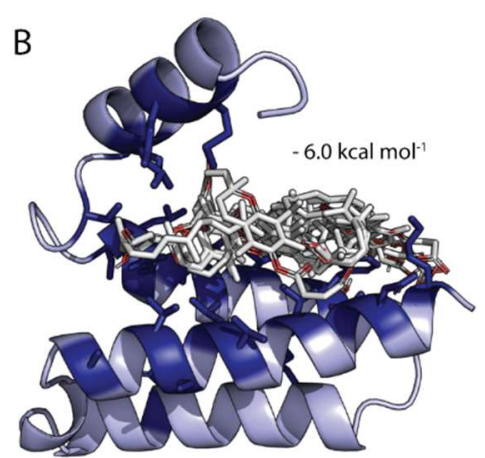

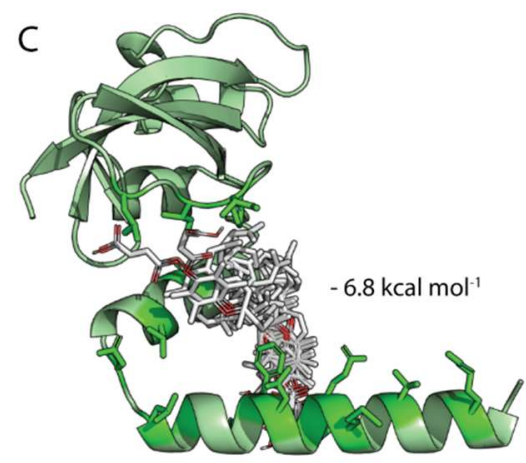

$\mathrm{E}$

$$
\begin{aligned}
& \text { Pose NSP7 NSP8 NSP12 } \\
& \begin{array}{l|lll}
\hline 1 & -6.0 & -6.8 & -6.7
\end{array} \\
& \begin{array}{l|lll}
2 & -6.0 & -6.3 & -6.4
\end{array} \\
& \begin{array}{l|lll}
\hline 3 & -6.0 & -6.3 & -6.3
\end{array} \\
& \begin{array}{l|lll}
4 & -5.9 & -6.3 & -6.3
\end{array} \\
& \begin{array}{l|lll}
5 & -5.8 & -6.2 & -6.2
\end{array} \\
& \begin{array}{l|lll}
6 & -5.8 & -6.2 & -6.2
\end{array} \\
& \begin{array}{l|lll}
7 & -5.7 & -6.2 & -6.2
\end{array} \\
& \begin{array}{l|lll}
8 & -5.7 & -6.1 & -6.1
\end{array} \\
& \begin{array}{l|lll}
9 & -5.7 & -6.1 & -6.0
\end{array} \\
& \begin{array}{l|lll}
10 & -5.7 & -6.1 & -6.0 \\
\hline
\end{array}
\end{aligned}
$$

Figure S8. Binding of $\alpha$ TOS to conserved, hydrophobic interfaces in the SARS-CoV-2 RdRp is energetically favorable. A) Docking model of the ten most favorable poses for aTOS (white) interacting with NSP12 (light red). B) Docking model of the ten most favorable poses for aTOS (white) interacting with conserved, hydrophobic residues (dark blue) within NSP7 (light blue). C) Docking model of the ten most favorable poses for aTOS (white) interacting with conserved, hydrophobic residues (dark green) within NSP7 (light green). D) Representation of the heterotetrameric structure of NSP7 and NSP8 with the most favorable poses of aTOS (opaque) interacting individually with each NSP7 (transparent, blue) and NSP 8 (transparent, green) superimposed. E) Table of the top ten poses for $\alpha$ TOS interacting with NSP7, NSP8, and NSP12. Values represent the predicted binding affinity in $\mathrm{kcal} / \mathrm{mol}$. 